\title{
INTERPOLATING SEQUENCES ON ANALYTIC BESOV TYPE SPACES
}

\author{
NICOLA ARCOZZI, DANIEL BLASI, AND JORDI PAU
}

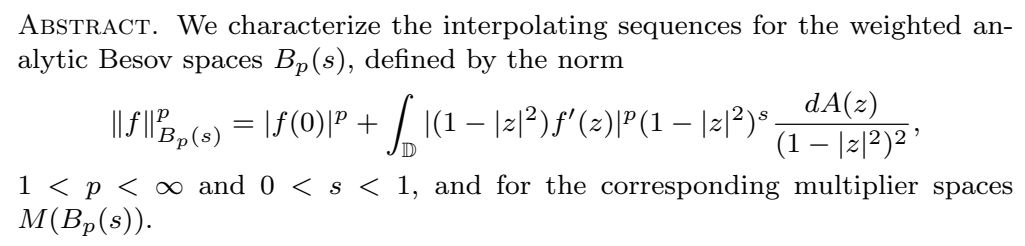

\section{Contents}

1. Introduction and main results

2. Background and preliminaries

3. Carleson measures and multipliers for $B_{p}(s)$

4. Boundary values

5. Necessity of condition (CS)

6. A $\bar{\partial}$-problem with estimates and the corona theorem

7. Sufficiency of condition (CS)

8. Concluding remarks 23

$\begin{array}{ll}\text { References } & 27\end{array}$

\section{InTRODUCTION AND MAIN RESUlts}

In this paper we characterize the interpolating sequences for the multiplier spaces of the family of weighted Besov spaces $B_{p}(s), 1<p<\infty, 0<s<1$, defined below. Throughout the paper, we denote by $M(X)$ the multiplier space of the Banach space $X$. The problem of finding the interpolating sequences for spaces of holomorphic functions (and their multiplier spaces) is an old one. The prototype of all such results is Carleson's celebrated Interpolation Theorem [8] for $H^{\infty}=M\left(H^{p}\right)$, later extended to the spaces $H^{p}$ themselves, $0<p<\infty$ in [24],[17]. All known proofs of Carleson's Theorem in the Hardy class make an essential use of Blaschke products, and this has been so far the main obstruction to an extension of the theorem to

2000 Mathematics Subject Classification. 30H05, 31C25, 46J15.

Key words and phrases. Besov spaces, interpolating sequences, Carleson measures, corona problems.

The first author is partially supported by the COFIN project Analisi Armonica, funded by the Italian Minister for Research. The second and third authors are supported by the grant 2005SGR00774. Also, the second author is partially supported by the DGICYT grant MTM200500544, while the third author is partially supported by the DGICYT grant MTM2005-08984-C0202. 
several complex variables. Note that $H^{2}=B_{2}(1)$ falls just outside the range of spaces here considered. Another result which is relevant to the present paper is that concerning the multiplier space $M\left(B_{2}(0)\right)$ of the Dirichlet space $B_{2}(0)$, whose interpolating sequences were characterized in the very deep paper [19] (see also [3]). The extension to $M\left(B_{p}(0)\right), 1<p<\infty$, is far from trivial and it is carried out in [6]. In the $s=0$ case the Blaschke products play no role, which does not come as a surprise because, for instance, $B_{2}(0)$, hence $M\left(B_{2}(0)\right)$, does not contain infinite Blaschke products. There is another instance in which the space $B_{2}(0)$ differs from $B_{2}(1)$. The Carleson measures for $B_{2}(1)=H^{2}$ (first characterized in [9]) are characterized by a simple condition to be checked on single boxes, while, when $0<s \leq 1$, characterizing the Carleson measures for $B_{p}(s)$ requires more complicated conditions, see [29], [30], [1].

Our characterization of the interpolating sequences for $M\left(B_{p}(s)\right)$ relies on the fact that, for $0<s<1$, the space $M\left(B_{p}(s)\right)$ is rich of Blaschke products, hence it is possible to mimic many arguments from the Hardy endpoint $s=1$. On the other hand, the Carleson measures are more similar to those of the Dirichlet endpoint $s=0$, and this accounts for some involved proofs.

The universal interpolating sequences for the spaces $B_{p}(s), 1<p<\infty, 0<s<$ 1 , were characterized in [11], a paper that did not receive the attention it deserved. An alternative proof easily follows from our construction of the interpolating sequences for $M\left(B_{p}(s)\right)$, and it is given in this paper. The characterization of the interpolating sequences for $M\left(B_{2}(s)\right)$ is also studied in [31]. A beautiful, short proof for $M\left(B_{2}(s)\right)$, which heavily relies on the Hilbert space nature of $B_{2}(s)$, is given in [7]. In the proof, we follow the basic ideas in [15] and [16], by constructing an explicit solution of an auxiliary $\bar{\partial}$-problem.

Main results. For $1<p<\infty$ and $0 \leq s<1$, let $B_{p}(s)$ be the Besov type space of those analytic functions on the unit disc $\mathbb{D}$ for which

$$
\|f\|_{B_{p}(s)}=\left(\int_{\mathbb{D}}\left|f^{\prime}(z)\right|^{p} d A_{p, s}(z)\right)^{1 / p}+|f(0)|<\infty
$$

where $d A_{p, s}(z)=\left(1-|z|^{2}\right)^{p-2+s} d A(z)$, and $d A$ is the normalized area measure.

The reproducing formula for $B_{p}(s)$ (see [5] or [22] for the case $p=2$ ) gives

$$
f(z)=f(0)+\int_{\mathbb{D}} f^{\prime}(w) K(z, w)\left(1-|w|^{2}\right)^{s} d A(w),
$$

where

$$
K(z, w)=\frac{1-(1-\bar{w} z)^{1+s}}{\bar{w}(1-\bar{w} z)^{1+s}} .
$$

From (1.1) it is easy to deduce that the point evaluations are bounded linear functionals in $B_{p}(s)$. Let $w(z)$ denote the norm of the point-evaluation functional at the point $z$. Then a sequence $Z=\left\{z_{n}\right\}$ of distinct points of $\mathbb{D}$ is called a universal interpolating sequence for the Besov type space $B_{p}(s)$ if $f \mapsto\left\{\frac{f\left(z_{n}\right)}{w\left(z_{n}\right)}\right\}$ maps $B_{p}(s)$ into and onto $l^{p}$.

The (pointwise) multipliers of $B_{p}(s)$ denoted by $M\left(B_{p}(s)\right)$ are those analytic functions $g$ for which $g f$ is in $B_{p}(s)$ whenever $f$ is in $B_{p}(s)$. The algebra $M\left(B_{p}(s)\right)$ plays a role in the study of the space $B_{p}(s)$ which is similar to the role played by $H^{\infty}$ in the study of the classical Hardy spaces $H^{p}$. The corresponding notion 
of interpolating sequences for such functions is that the map $f \mapsto\left\{f\left(z_{n}\right)\right\}$ maps $M\left(B_{p}(s)\right)$ onto $l^{\infty}$.

As for bounded analytic functions (see [8]), the characterization of the interpolating sequences involves a separation condition that says how close two points in the sequence can be. A sequence $\left\{z_{n}\right\}$ of points of $\mathbb{D}$ is called a separated sequence if

$$
\inf _{j \neq k} \rho\left(z_{j}, z_{k}\right)>0
$$

where $\rho$ is the pseudohyperbolic distance

$$
\rho(z, w)=\left|\frac{z-w}{1-\bar{w} z}\right| .
$$

This condition is equivalent to the fact that there is a positive constant $\delta<1$ such that the pseudohyperbolic discs $D_{z_{j}}=\left\{z: \rho\left(z, z_{j}\right)<\delta\right\}$ are pairwise disjoints.

As for the classical Hardy spaces $H^{p}$, a positive Borel measure $\mu$ on $\mathbb{D}$ is called an $(s, p)$-Carleson measure if there is a positive constant $C$ such that

$$
\int_{\mathbb{D}}|f|^{p} d \mu \leq C\|f\|_{B_{p}(s)}^{p}
$$

whenever $f$ is in $B_{p}(s)$. The role of these measures in the characterization of interpolating sequences for $B_{p}(s)$ is similar to the $H^{p}$ situation.

Throughout the paper, $q$ will denote the conjugate exponent of $p$, that is, $\frac{1}{p}+\frac{1}{q}=1$. We can now state our main theorem.

Theorem 1. Let $1<p<\infty$ and $0<s<1$. The following are equivalent for a sequence $Z=\left\{z_{n}\right\}$ in $\mathbb{D}$.

(M) $Z$ is an interpolating sequence for $M\left(B_{p}(s)\right)$.

(RS) The following norm equivalence holds:

$$
\left\|\sum_{j} a_{j} \frac{k_{z_{j}}}{\left\|k_{z_{j}}\right\|_{B_{q}(s)}}\right\|_{B_{q}(s)} \asymp\left(\sum_{j}\left|a_{j}\right|^{q}\right)^{1 / q} .
$$

(UIS) $Z$ is an universal interpolating sequence for $B_{p}(s)$.

(CS) $Z$ is a separated sequence and $\mu_{Z}=\sum_{z_{n} \in Z}\left(1-\left|z_{n}\right|^{2}\right)^{s} \delta_{z_{n}}$ is an $(s, p)$ Carleson measure.

We point out that in the case $p=2$, the equivalence between (CS) and (UIS) was proved by Cohn in [11]. Note that, since the real part of the derivative of the reproducing kernels is not always positive, we can not use the generalization of the standard Hilbert space techniques developed in [6] to prove that condition (M) implies conditions (RS) and (UIS). Instead of that, since it is standard that conditions (RS) and (UIS) are equivalent (see, e.g. [2, p.42]), we will show that condition (CS) is necessary and sufficient for both the multiplier and the universal interpolation problems.

The paper is organized as follows: Section 2 is devoted to background material and preliminaries for the analytic Besov-type spaces $B_{p}(s)$. In Section 4 we prove some relations between boundary value functions $f \in L_{s}^{p}$ and some extensions in $\mathbb{D}$, and we also study the multipliers of $L_{s}^{p}$ in terms of $(s, p)$-Carleson measures. In Section 5 we prove the necessity part of Theorem 1 . In Section 6 , we study a $\bar{\partial}$-problem with estimates needed to prove the sufficiency part in Section 7 . This 
$\bar{\partial}$-problem is also applied to prove the corona theorem for the multiplier algebra. $f$,

Also, given a function $f \in L^{1}(\mathbb{T})$, we denote by $\widehat{f}$ to be the Poisson integral of

$$
\widehat{f}(z)=\frac{1}{2 \pi} \int_{0}^{2 \pi} f\left(e^{i \theta}\right) \frac{1-|z|^{2}}{\left|e^{i \theta}-z\right|^{2}} d \theta .
$$

The letter $C$ will denote an absolute constant whose value may change from line to line. We also use the notation $a \lesssim b$ to indicate that there is a constant $C>0$ with $a \leq C b$, and the notation $a \asymp b$ means that $a \lesssim b$ and $b \lesssim a$.

\section{BACKGROUND AND PRELIMINARIES}

Consider the inner product

$$
\langle f, g\rangle_{s}=f(0) \overline{g(0)}+\int_{\mathbb{D}} f^{\prime}(z) \overline{g^{\prime}(z)}\left(1-|z|^{2}\right)^{s} d A(z)
$$

for $f \in B_{p}(s)$ and $g \in B_{q}(s)$. By Hölder's inequality,

$$
\left|\langle f, g\rangle_{s}\right| \leq\|f\|_{B_{p}(s)}\|g\|_{B_{q}(s)}
$$

for $f \in B_{p}(s)$ and $g \in B_{q}(s)$. With that pairing, the dual of $B_{p}(s)$ can be identified with $B_{q}(s)$ (see Lemma 2.1 below). Since the point-evaluations are continuous, there are reproducing kernels $k_{z} \in B_{q}(s)$ with $f(z)=\left\langle f, k_{z}\right\rangle_{s}$, and it is easy to compute that $w(z) \approx\left\|k_{z}\right\|_{B_{q}(s)} \approx\left(1-|z|^{2}\right)^{-s / p}$.

The following lemma is proved in Section 4.2 of [32].

Lemma A. Suppose $z \in \mathbb{D}, c>0$ and $t>-1$, then the integral

$$
I_{c, t}(z)=\int_{\mathbb{D}} \frac{\left(1-|w|^{2}\right)^{t}}{|1-\bar{w} z|^{2+t+c}} d A(w)
$$

is comparable to $\left(1-|z|^{2}\right)^{-c}$.

The next result is taken from [5].

Lemma B. Let $1<p<\infty$, and let $\sigma>-1 b \geq 0$ with $b<2+\sigma$. Let $g$ be analytic on $\mathbb{D}$. Then,

$$
\int_{\mathbb{D}}|g(z)-g(0)|^{p} \frac{\left(1-|z|^{2}\right)^{\sigma}}{|1-\bar{w} z|^{b}} d A(z) \leq C \int_{\mathbb{D}}\left(1-|z|^{2}\right)^{p}\left|g^{\prime}(z)\right|^{p} \frac{\left(1-|z|^{2}\right)^{\sigma}}{|1-\bar{w} z|^{b}} d A(z) .
$$

The following is standard and can be proved as other duality results in [32].

Lemma 2.1. Let $1<p<\infty$ and $0 \leq s<1$. The dual space of $B_{p}(s)$ can be identified with $B_{q}(s)$ (with equivalent norms) under the pairing

$$
\langle f, g\rangle_{s}=f(0) \overline{g(0)}+\int_{\mathbb{D}} f^{\prime}(z) \overline{g^{\prime}(z)}\left(1-|z|^{2}\right)^{s} d A(z),
$$

where $f \in B_{p}(s)$ and $g \in B_{q}(s)$.

Let $k_{z} \in B_{q}(s)$ be the reproducing kernel associated with the pairing $<,>_{s}$. From (1.1) it follows that $k_{z}(0)=1$ and

$$
k_{z}^{\prime}(\zeta)=\frac{1}{\zeta} \frac{1-(1-\bar{z} \zeta)^{1+s}}{(1-\bar{z} \zeta)^{1+s}} .
$$

Now, we state some properties of the reproducing kernels $k_{z}$. 
Lemma 2.2. Let $1<p<\infty$, and $s>0$. Then

$$
\left\|k_{z}\right\|_{B_{q}(s)}^{p} \asymp\left(1-|z|^{2}\right)^{-s} .
$$

Proof. This follows by direct calculation using the above expression for the derivative of the reproducing kernel and Lemma A.

Lemma 2.3. Let $1<p<\infty$, and $s>0$. Let $z, w \in \mathbb{D}$ having pseudo-hyperbolic distance $\rho(z, w) \leq c<1$. Then

$$
\left\|k_{z}-k_{w}\right\|_{B_{q}(s)} \leq C\left|\frac{z-w}{1-\bar{w} z}\right|\left(1-|w|^{2}\right)^{-s / p}
$$

Proof. By the mean value theorem, there exists $\nu \in[z, w]$, the line segment joining $z$ and $w$, such that for $\zeta \in \mathbb{D}$,

$$
\left|(1-\bar{w} \zeta)^{1+s}-(1-\bar{z} \zeta)^{1+s}\right| \leq(1+s)|\zeta||1-\bar{\nu} \zeta|^{s}|z-w| .
$$

Using this, we obtain

$$
\left|k_{z}^{\prime}(\zeta)-k_{w}^{\prime}(\zeta)\right|=\left|\frac{(1-\bar{z} \zeta)^{1+s}-(1-\bar{w} \zeta)^{1+s}}{\zeta(1-\bar{w} \zeta)^{1+s}(1-\bar{z} \zeta)^{1+s}}\right| \lesssim \frac{|z-w||1-\bar{\nu} \zeta|^{s}}{|1-\bar{w} \zeta|^{1+s}|1-\bar{z} \zeta|^{1+s}} .
$$

Since $\rho(z, w) \leq c<1$, then $|1-\bar{\nu} \zeta| \asymp|1-\bar{w} \zeta| \asymp|1-\bar{z} \zeta|$, and we get

$$
\left|k_{z}^{\prime}(\zeta)-k_{w}^{\prime}(\zeta)\right| \lesssim \frac{|z-w|}{|1-\bar{w} \zeta|^{2+s}}
$$

Now, using (2.2) and Lemma A, we obtain

$$
\begin{aligned}
\left\|k_{z}-k_{w}\right\|_{B_{q}(s)} & =\left(\int_{\mathbb{D}}\left|k_{z}^{\prime}(\zeta)-k_{w}^{\prime}(\zeta)\right|^{q}\left(1-|\zeta|^{2}\right)^{q-2+s} d A(\zeta)\right)^{1 / q} \\
& \lesssim|z-w|\left(\int_{\mathbb{D}} \frac{\left(1-|\zeta|^{2}\right)^{q-2+s} d A(\zeta)}{|1-\bar{w} \zeta|^{2 q+q s}}\right)^{1 / q} \\
& \lesssim \frac{|z-w|}{1-|w|^{2}}\left(1-|w|^{2}\right)^{-\frac{(q-1) s}{q}} \\
& \asymp\left|\frac{z-w}{1-\bar{w} z}\right|\left(1-|w|^{2}\right)^{-s / p} .
\end{aligned}
$$

The proof of the necessity part of condition (CS) in Theorem 1 uses a randomization technique that has been very useful in many other areas of analysis. Thus we are going to discuss Khinchine's inequality. Given finitely many complex numbers $\alpha_{1}, \ldots, \alpha_{n}$, consider the $2^{n}$ possible sums

$$
\sum_{j=1}^{n} \pm \alpha_{j}
$$

obtained as the plus-minus signs vary in the $2^{n}$ possible ways. For $r>0$ we use

$$
\mathcal{E}\left(\left|\sum_{j=1}^{n} \pm \alpha_{j}\right|^{r}\right)
$$


to denote the average value of $\left|\sum_{j=1}^{n} \pm \alpha_{j}\right|^{r}$ over the $2^{n}$ choices of sign. To be precise, let $\Omega$ be the set of $2^{n}$ points $\omega=\left(\omega_{1}, \omega_{2}, \ldots, \omega_{n}\right)$, where $\omega_{j}= \pm 1$. Define the probability $\mu$ on $\Omega$ so that each point $\omega$ has probability $2^{-n}$. Also define

$$
X(\omega)=\sum_{j=1}^{n} \alpha_{j} \omega_{j} .
$$

Then $X(\omega)$ is a more rigorous expression for $\sum \pm \alpha_{j}$, and by definition

$$
\mathcal{E}\left(\left|\sum_{j=1}^{n} \pm \alpha_{j}\right|^{r}\right)=\frac{1}{2^{n}} \sum_{\omega \in \Omega}|X(\omega)|^{p}=\int_{\Omega}|X(\omega)|^{p} d \mu .
$$

Khinchine's inequality. Let $r>0$, then

$$
\mathcal{E}\left(\left|\sum_{j=1}^{n} \pm \alpha_{j}\right|^{r}\right) \leq C_{r}\left(\sum_{j=1}^{n}\left|\alpha_{j}\right|^{2}\right)^{r / 2}
$$

(see [13, p. 302]). Actually $C_{r}=1$ if $r \leq 2$. The important thing in (2.3) is that $C_{r}$ does not increase as $n$ increases. This inequality will be used in the reproducing formula for $B_{p}(s)$. We also use the elementary inequalities

$$
\left(\mathcal{E}\left|\sum_{k=1}^{n} \pm a_{k}\right|\right)^{t} \leq \mathcal{E}\left(\left|\sum_{k=1}^{n} \pm a_{k}\right|^{t}\right), \quad t \geq 1
$$

and

$$
\sum\left|a_{k}\right| \leq\left(\sum\left|a_{k}\right|^{r}\right)^{1 / r}, \quad 0<r \leq 1
$$

\section{Carleson measures and multipliers for $B_{p}(s)$}

Given an $\operatorname{arc} I$ of the unit circle $\mathbb{T}$ of normalized arclength $|I|$, consider the Carleson box $S(I)$ defined as

$$
S(I)=\left\{r e^{i t} \in \mathbb{D}: 1-r \leq|I| ; e^{i t} \in I\right\} .
$$

Let $s>0$. A positive Borel measure on $\mathbb{D}$ is said to be an $s$-Carleson measure if

$$
\sup _{I \subset \mathbb{T}} \frac{\mu(S(I))}{|I|^{s}}<\infty .
$$

When $s=1$ we obtain the classical Carleson measures, that by Carleson embedding theorem, are just the measures characterizing when the embedding $H^{p} \subset L^{p}(\mu)$ is continuous.

A positive Borel measure on $\mathbb{D}$ is a Carleson measure for $B_{p}(s)$ (or an $(s, p)$ Carleson measure) if the embedding $B_{p}(s) \subset L^{p}(\mu)$ is continuous, that is, if there is a positive constant $C$ such that

$$
\int_{\mathbb{D}}|f(z)|^{p} d \mu(z) \leq C\|f\|_{B_{p}(s)}^{p}
$$

whenever $f$ is in $B_{p}(s)$. There are several characterizations of Carleson measures for $B_{p}(s)$ (see [29] and [30] for a capacitary condition analogous to Stegenga's description in [25] of Carleson measures for $B_{p}$, or [1] and [18] for non capacitary 
conditions), but for our purposes we only need the following simple observation proved in [5].

Lemma C. Let $1<p<\infty$ and $0 \leq s \leq 1$. Let $\mu$ be a Carleson measure for $B_{p}(s)$. Then for each $\varepsilon>0$

$$
\sup _{a \in \mathbb{D}} \int_{\mathbb{D}} \frac{\left(1-|a|^{2}\right)^{\varepsilon}}{|1-\bar{a} z|^{\varepsilon+s}} d \mu(z) \leq C,
$$

where $C$ is a positive constant independent of $\varepsilon$.

The following is a well known generalization of the characterization of classical Carleson measures in conformal invariant terms. See [13, Chapter VI, Lemma 3.3] for the classical case and [4, Lemma 4.1] for the general case.

Lemma D. Let $s>0$. Then $\mu$ is an $s$-Carleson measure if and only if

$$
\sup _{a \in \mathbb{D}} \int_{\mathbb{D}}\left(\frac{1-|a|^{2}}{|1-\bar{a} z|^{2}}\right)^{s} d \mu(z)<\infty .
$$

Not all $s$-Carleson measures are Carleson measures for $B_{p}(s)$. It is known that the condition (3.1) is not sufficient for a measure $\mu$ to be a Carleson measure for $B_{p}(s)$ (see for example [19] for the case $p=2$ ), but it turns to be an important necessary condition.

Lemma 3.1. Let $1<p<\infty$, and $s>0$. If $\mu$ is a Carleson measure for $B_{p}(s)$, then $\mu$ is an $s$-Carleson measure.

Proof. This follows immediately after an application of Lemma $\mathrm{C}$ with $\varepsilon=s$, and then Lemma D.

We also need the following result (see [5]) that says that Carleson measures for $B_{p}(s)$ are stable under the action of some integral operator.

Lemma E. Let $0 \leq s<1,1<p<\infty$, and let

$$
T v(z)=\int_{\mathbb{D}} \frac{v(w)}{|1-\bar{w} z|^{2}} d A(z) .
$$

If $|v(z)|^{p} d A_{p, s}(z)$ is a Carleson measure for $B_{p}(s)$ (and $|v(z)|(1-|z|) \leq C$ when $1<p<2)$, then $|T v(z)|^{p} d A_{p, s}(z)$ is also a Carleson measure for $B_{p}(s)$.

Recall that the algebra $M\left(B_{p}(s)\right)$ of pointwise multipliers of $B_{p}(s)$, consists of those analytic functions on $\mathbb{D}$ such that $g f \in B_{p}(s)$ whenever $f \in B_{p}(s)$. Identifying a multiplier $g$ with the operator given by $M_{g}(f)=g f$, and using the operator norm as the multiplier norm, $M\left(B_{p}(s)\right)$ becomes a Banach algebra.

The following well known result (see, e.g. [1, p.472]) describes the multipliers of $B_{p}(s)$ in terms of $(s, p)$-Carleson measures.

Lemma F. Let $1<p<\infty$ and $0 \leq s<1$. Then $f \in M\left(B_{p}(s)\right)$ if and only if $f \in H^{\infty}$ and $\left|f^{\prime}(z)\right|^{p} d A_{p, s}(z)$ is an $(s, p)$-Carleson measure.

The next result says that there are infinite Blaschke products in $M\left(B_{p}(s)\right)$. This is proved in [29]. We give an elementary proof of it to keep the paper self-contained. 
Proposition 3.2. Let $1<p<\infty$ and $0<s<1$. Let $\left\{z_{n}\right\}$ be a sequence in $\mathbb{D}$ such that $\sum_{n}\left(1-\left|z_{n}\right|^{2}\right)^{s} \delta_{z_{n}}$ is an $(s, p)$-Carleson measure, then $B$ belongs to the multiplier algebra $M\left(B_{p}(s)\right)$, where

$$
B(z)=\prod_{n} \frac{\left|z_{n}\right|}{z_{n}} \frac{z_{n}-z}{1-\bar{z}_{n} z}
$$

is the Blaschke product with zeros $\left\{z_{n}\right\}$.

Proof. Note first that the assumption also implies that the measure

$$
\mu_{\left\{z_{n}\right\}}=\sum_{n}\left(1-\left|z_{n}\right|^{2}\right) \delta_{z_{n}}
$$

is a 1-Carleson measure. Indeed, let $S(I)$ be a Carleson box, then

$$
\mu_{\left\{z_{n}\right\}}(S(I))=\sum_{z_{n} \in S(I)}\left(1-\left|z_{n}\right|^{2}\right) \leq|I|^{1-s} \sum_{z_{n} \in S(I)}\left(1-\left|z_{n}\right|^{2}\right)^{s} \leq C|I| .
$$

For $a \in \mathbb{D}$, let $\varphi_{a}(z)=\frac{a-z}{1-\bar{a} z}$. By Lemma $\mathrm{D}$ with $s=1$, for all $z \in \mathbb{D}$ we have

$$
\sum_{n}\left(1-\left|\varphi_{z_{n}}(z)\right|^{2}\right)=\int_{\mathbb{D}}\left(\frac{1-|z|^{2}}{|1-\bar{w} z|^{2}}\right) d \mu_{\left\{z_{n}\right\}}(w) \leq C .
$$

By Lemma $\mathrm{F}$, the condition that $B \in M\left(B_{p}(s)\right)$ is equivalent to the fact that $\left|B^{\prime}(z)\right|^{p} d A_{p, s}(z)$ is an $(s, p)$-Carleson measure. Let $g \in B_{p}(s)$. Since $B \in H^{\infty} \subset \mathcal{B}$, the Bloch space, we have

$$
\left(1-|z|^{2}\right)\left|B^{\prime}(z)\right| \leq C
$$

So, by (3.4), and using the estimate

$$
\left|B^{\prime}(z)\right| \leq \sum_{n} \frac{\left(1-\left|z_{n}\right|^{2}\right)}{\left|1-\bar{z}_{n} z\right|^{2}}
$$

we have

$$
\begin{aligned}
\int_{\mathbb{D}}|g(z)|^{p}\left|B^{\prime}(z)\right|^{p} d A_{p, s}( & z) \lesssim \int_{\mathbb{D}}|g(z)|^{p}\left(\sum_{n} \frac{1-\left|z_{n}\right|^{2}}{\left|1-\bar{z}_{n} z\right|^{2}}\right)\left(1-|z|^{2}\right)^{s-1} d A(z) \\
= & \sum_{n}\left(1-\left|z_{n}\right|^{2}\right) \int_{\mathbb{D}}|g(z)|^{p} \frac{\left(1-|z|^{2}\right)^{s-1}}{\left|1-\bar{z}_{n} z\right|^{2}} d A(z) \\
& \lesssim \sum_{n}\left(1-\left|z_{n}\right|^{2}\right) \int_{\mathbb{D}}\left|g\left(z_{n}\right)\right|^{p} \frac{\left(1-|z|^{2}\right)^{s-1}}{\left|1-\bar{z}_{n} z\right|^{2}} d A(z) \\
& +\sum_{n}\left(1-\left|z_{n}\right|^{2}\right) \int_{\mathbb{D}}\left|g(z)-g\left(z_{n}\right)\right|^{p} \frac{\left(1-|z|^{2}\right)^{s-1}}{\left|1-\bar{z}_{n} z\right|^{2}} d A(z) \\
& =I_{1}+I_{2} .
\end{aligned}
$$

By Lemma A and the fact that $\sum_{n}\left(1-\left|z_{n}\right|^{2}\right)^{s} \delta_{z_{n}}$ is an $(s, p)$-Carleson measure, we have

$$
\begin{aligned}
I_{1} & =\sum_{n}\left|g\left(z_{n}\right)\right|^{p}\left(1-\left|z_{n}\right|^{2}\right) \int_{\mathbb{D}} \frac{\left(1-|z|^{2}\right)^{s-1}}{\left|1-\bar{z}_{n} z\right|^{2}} d A(z) \\
& \lesssim \sum_{n}\left|g\left(z_{n}\right)\right|^{p}\left(1-\left|z_{n}\right|^{2}\right)^{s} \lesssim\|g\|_{B_{p}(s)}^{p} .
\end{aligned}
$$


On the other hand, making the change of variables $w=\varphi_{z_{n}}(z)$ we have ${ }^{1}$

$$
\begin{aligned}
I_{2} & =\sum_{n}\left(1-\left|z_{n}\right|^{2}\right) \int_{\mathbb{D}}\left|g(z)-g\left(z_{n}\right)\right|^{p} \frac{\left(1-|z|^{2}\right)^{s-1}}{\left|1-\bar{z}_{n} z\right|^{2}} d A(z) \\
& =\sum_{n}\left(1-\left|z_{n}\right|^{2}\right)^{s} \int_{\mathbb{D}}\left|g_{z_{n}}(w)-g_{z_{n}}(0)\right|^{p} \frac{\left(1-|w|^{2}\right)^{s-1}}{\left|1-\bar{z}_{n} w\right|^{2 s}} d A(w),
\end{aligned}
$$

where $g_{z_{n}}=g \circ \varphi_{z_{n}}$. Therefore, since $s-1>-1$ and $2 s<1+s$, by Lemma B, a change of variables $z=\varphi_{z_{n}}(w)$, and (3.3) we get

$$
\begin{aligned}
I_{2} & \lesssim \sum_{n}\left(1-\left|z_{n}\right|^{2}\right)^{s} \int_{\mathbb{D}}\left|\left(g \circ \varphi_{z_{n}}\right)^{\prime}(w)\right|^{p} \frac{\left(1-|w|^{2}\right)^{p+s-1}}{\left|1-\bar{z}_{n} w\right|^{2 s}} d A(w) \\
& =\sum_{n} \int_{\mathbb{D}}\left|g^{\prime}(z)\right|^{p}\left(1-\left|\varphi_{z_{n}}(z)\right|^{2}\right) d A_{p, s}(z) \leq C\|g\|_{B_{p}(s)}^{p} .
\end{aligned}
$$

So, putting together (3.5), (3.6) and (3.7) the proof is completed.

\section{Boundary values}

Since for $s<1, B_{p}(s) \subset H^{p}$, it follows that every function $f$ in $B_{p}(s)$ has nontangential limits a.e. on $\mathbb{T}$. Denote by $f_{b}$ the boundary values of $f$ (taken as a nontangential limit). The purpose of this section is to give a description of the space $B_{p}(s)$ in terms of its boundary values.

Let $f \in L^{p}(\mathbb{T})$. We say that $f \in L_{s}^{p}$ if the seminorm

$$
\|f\|_{L_{s}^{p}}^{* p}=\int_{0}^{2 \pi} \int_{0}^{2 \pi} \frac{\left|f\left(e^{i t}\right)-f\left(e^{i u}\right)\right|^{p}}{\left|e^{i t}-e^{i u}\right|^{2-s}} d u d t
$$

is finite. A norm for this space can be defined as $\|f\|_{L_{s}^{p}}^{p}=\|f\|_{L_{s}^{p}}^{* p}+\|f\|_{L^{p}(\mathbb{T})}^{p}$.

Lemma 4.1. Let $1<p<\infty$ and $0<s<1$. Let $f \in L^{p}(\mathbb{T})$ and let $F \in C^{1}(\mathbb{D})$ with $\lim _{r \rightarrow 1} F\left(r e^{i \theta}\right)=f\left(e^{i \theta}\right)$ for a.e. $e^{i \theta} \in \mathbb{T}$. If $\int_{\mathbb{D}}|\nabla F(z)|^{p} d A_{p, s}(z)<\infty$ then $f \in L_{s}^{p}$.

Proof. It is enough to show that

$$
\|f\|_{L_{s}^{p}}^{* p}=\int_{0}^{2 \pi} \int_{0}^{2 \pi} \frac{\left|f\left(e^{i t}\right)-f\left(e^{i u}\right)\right|^{p}}{\left|e^{i t}-e^{i u}\right|^{2-s}} d u d t \lesssim \int_{\mathbb{D}}|\nabla F(z)|^{p} d A_{p, s}(z) .
$$

To see that, we use an argument from [20]. Changing the coordinates we obtain

$$
\begin{aligned}
\|f\|_{L_{s}^{p}}^{* p} & =\int_{0}^{2 \pi} \frac{1}{\left|e^{i \varphi}-1\right|^{2-s}}\left(\int_{0}^{2 \pi}\left|f\left(e^{i(u+\varphi)}\right)-f\left(e^{i u}\right)\right|^{p} d u\right) d \varphi \\
& \leq C \int_{0}^{1 / 2} \frac{1}{h^{2-s}} \int_{0}^{2 \pi}\left|f\left(e^{i(u+h)}\right)-f\left(e^{i u}\right)\right|^{p} d u d h .
\end{aligned}
$$

\footnotetext{
${ }^{1}$ Recall that

$$
\varphi_{a}^{\prime}(z)=\frac{1-\left|\varphi_{a}(z)\right|^{2}}{1-|z|^{2}}, \quad 1-\left|\varphi_{a}(z)\right|^{2}=\frac{\left(1-|a|^{2}\right)\left(1-|z|^{2}\right)}{|1-\bar{a} z|^{2}}, \quad \varphi_{a} \circ \varphi_{a}=I d .
$$
}


Take $r=1-h$. We have

$$
\begin{aligned}
\left|f\left(e^{i(u+h)}\right)-f\left(e^{i u}\right)\right| \leq & \left|f\left(e^{i(u+h)}\right)-F\left(r e^{i(u+h)}\right)\right|+\left|F\left(r e^{i(u+h)}\right)-F\left(r e^{i u}\right)\right| \\
& +\left|F\left(r e^{i u}\right)-f\left(e^{i u}\right)\right| \\
\leq & \int_{r}^{1}\left|\nabla F\left(x e^{i(u+h)}\right)\right| d x+\int_{0}^{h}\left|\nabla F\left(r e^{i(u+t)}\right)\right| d t \\
& +\int_{r}^{1}\left|\nabla F\left(x e^{i u}\right)\right| d x .
\end{aligned}
$$

Apply Minkowski integral inequality [26, p. 271], to get

$$
\begin{aligned}
\int_{0}^{2 \pi} \mid f\left(e^{i(u+h)}\right) & -\left.f\left(e^{i u}\right)\right|^{p} d u \\
& \lesssim \int_{0}^{2 \pi}\left(\int_{r}^{1}\left|\nabla F\left(x e^{i u}\right)\right| d x\right)^{p} d u+\int_{0}^{2 \pi}\left(\int_{0}^{h}\left|\nabla F\left(r e^{i(u+t)}\right)\right| d t\right)^{p} d u \\
& \lesssim\left(\int_{r}^{1}\left(\int_{0}^{2 \pi}\left|\nabla F\left(x e^{i u}\right)\right|^{p} d u\right)^{1 / p} d x\right)^{p} \\
& +\left(\int_{0}^{h}\left(\int_{0}^{2 \pi}\left|\nabla F\left(r e^{i(u+t)}\right)\right|^{p} d u\right)^{1 / p} d t\right)^{p}=(I)+(I I) .
\end{aligned}
$$

For the term $(I)$, after the change of variables $x=1-t$, we apply Hardy's inequality $[26$, p. 272] to obtain

$$
\begin{aligned}
\int_{0}^{1 / 2} \frac{1}{h^{2-s}}(I) d h & =\int_{0}^{1 / 2} \frac{1}{h^{2-s}}\left(\int_{r}^{1}\left(\int_{0}^{2 \pi}\left|\nabla F\left(x e^{i u}\right)\right|^{p} d u\right)^{1 / p} d x\right)^{p} d h \\
& =\int_{0}^{1 / 2} \frac{1}{h^{2-s}}\left(\int_{0}^{h}\left(\int_{0}^{2 \pi}\left|\nabla F\left((1-t) e^{i u}\right)\right|^{p} d u\right)^{1 / p} d t\right)^{p} d h \\
& \leq\left(\frac{p}{1-s}\right)^{p} \int_{0}^{1 / 2} \int_{0}^{2 \pi}\left|\nabla F\left((1-h) e^{i u}\right)\right|^{p} d u h^{p-2+s} d h \\
& \leq C \int_{\mathbb{D}}|\nabla F(z)|^{p} d A_{p, s}(z) .
\end{aligned}
$$

For the term $(I I)$ we just take $r=1-h$ and integrate with respect to $t$ and we obtain the desired result.

$$
\begin{aligned}
\int_{0}^{1 / 2} \frac{1}{h^{2-s}}(I I) d h & =\int_{0}^{1 / 2} \frac{1}{h^{2-s}}\left(\int_{0}^{h}\left(\int_{0}^{2 \pi}\left|\nabla F\left(r e^{i u}\right)\right|^{p} d u\right)^{1 / p} d t\right)^{p} d h \\
& =\int_{0}^{1 / 2} h^{p-2+s}\left(\int_{0}^{2 \pi}\left|\nabla F\left((1-h) e^{i u}\right)\right|^{p} d u\right) d h \\
& \leq C \int_{\mathbb{D}}|\nabla F(z)|^{p} d A_{p, s}(z) .
\end{aligned}
$$

So, we obtain (4.1), and the proof is finished. 
Proposition 4.2. Let $1<p<\infty, 0<s<1$, and let $f \in L^{p}(\mathbb{T})$. Then $f \in L_{s}^{p}$ if and only if

$$
\int_{\mathbb{D}}|\nabla \widehat{f}(z)|^{p} d A_{p, s}(z)<\infty
$$

where $\widehat{f}=P f$ is the Poisson integral of $f$. Moreover, there exists a universal constant $C$ such that

$$
C^{-1}\|f\|_{L_{s}^{p}}^{* p} \leq \int_{\mathbb{D}}|\nabla \widehat{f}(z)|^{p} d A_{p, s}(z) \leq C\|f\|_{L_{s}^{p}}^{* p}
$$

Proof. Consider $f \in L^{p}(\mathbb{T})$, and let $\widehat{f}$ be the Poisson integral of $f$. If

$$
\int_{\mathbb{D}}|\nabla \widehat{f}(z)|^{p} d A_{p, s}(z)<\infty
$$

then it follows from Lemma 4.1 that $f \in L_{s}^{p}$. For the converse we will show that

$$
\int_{\mathbb{D}}|\nabla \widehat{f}(z)|^{p} d A_{p, s}(z) \leq C \int_{0}^{2 \pi} \int_{0}^{2 \pi} \frac{\left|f\left(e^{i t}\right)-f\left(e^{i u}\right)\right|^{p}}{\left|e^{i t}-e^{i u}\right|^{2-s}} d u d t .
$$

Since

an easy computation gives

$$
\widehat{f}(z)=\int_{0}^{2 \pi} f\left(e^{i t}\right) \frac{\left(1-|z|^{2}\right)}{\left|e^{i t}-z\right|^{2}} \frac{d t}{2 \pi},
$$

$$
\left|\nabla \widehat{f}\left(r e^{i u}\right)\right| \leq C \int_{0}^{2 \pi} \frac{\left|f\left(e^{i t}\right)-f\left(e^{i u}\right)\right|}{\left|e^{i t}-r e^{i u}\right|^{2}} d t .
$$

Then, by Hölder's inequality

$$
\begin{aligned}
\left|\nabla \widehat{f}\left(r e^{i u}\right)\right|^{p} & \lesssim\left(\int_{0}^{2 \pi} \frac{\left|f\left(e^{i t}\right)-f\left(e^{i u}\right)\right|}{\left|e^{i t}-r e^{i u}\right|^{2}} d t\right)^{p} \\
& \leq \int_{0}^{2 \pi} \frac{\left|f\left(e^{i t}\right)-f\left(e^{i u}\right)\right|^{p}}{\left|e^{i t}-r e^{i u}\right|^{2}} d t\left(\int_{0}^{2 \pi} \frac{1}{\left|e^{i t}-r e^{i u}\right|^{2}} d t\right)^{p-1} \\
& =2 \pi\left(\int_{0}^{2 \pi} \frac{\left|f\left(e^{i t}\right)-f\left(e^{i u}\right)\right|^{p}}{\left|e^{i t}-r e^{i u}\right|^{2}} d t\right)\left(1-r^{2}\right)^{-(p-1)}
\end{aligned}
$$

So, using (4.3), we obtain

$$
\begin{aligned}
\int_{\mathbb{D}}|\nabla \widehat{f}(z)|^{p} & \left(1-|z|^{2}\right)^{p-2+s} d A(z) \\
& \lesssim \int_{0}^{2 \pi} \int_{0}^{1} \int_{0}^{2 \pi} \frac{\left|f\left(e^{i t}\right)-f\left(e^{i u}\right)\right|^{p}}{\left|e^{i t}-r e^{i u}\right|^{2}} d t\left(1-r^{2}\right)^{s-1} r d r d u \\
& =\int_{0}^{2 \pi} \int_{0}^{2 \pi}\left|f\left(e^{i t}\right)-f\left(e^{i u}\right)\right|^{p}\left(\int_{0}^{1} \frac{r\left(1-r^{2}\right)^{s-1}}{\left|e^{i t}-r e^{i u}\right|^{2}} d r\right) d t d u .
\end{aligned}
$$

So, the proof will be complete if we show

$$
\int_{0}^{1} \frac{r\left(1-r^{2}\right)^{s-1}}{\left|e^{i t}-r e^{i u}\right|^{2}} d r \lesssim \frac{1}{\left|e^{i t}-e^{i u}\right|^{2-s}}
$$

To prove (4.4), let $N$ be a positive integer with $2^{N-1}\left|e^{i t}-e^{i u}\right|<1<2^{N}\left|e^{i t}-e^{i u}\right|$, and set $R_{0}=\left\{r \in(0,1): 0<1-r \leq\left|e^{i t}-e^{i u}\right|\right\}$, and

$$
R_{n}=\left\{r \in(0,1): 2^{n-1}\left|e^{i t}-e^{i u}\right|<1-r \leq 2^{n}\left|e^{i t}-e^{i u}\right|\right\} \quad \text { for } n=1, \ldots, N \text {. }
$$


Using the fact that $\left|e^{i t}-r e^{i u}\right|^{2}=(1-r)^{2}+r\left|e^{i t}-e^{i u}\right|^{2}$, we get

$$
\int_{R_{0}} \frac{r\left(1-r^{2}\right)^{s-1}}{\left|e^{i t}-r e^{i u}\right|^{2}} d r \leq \frac{1}{\left|e^{i t}-e^{i u}\right|^{2}} \int_{R_{0}}(1-r)^{s-1} d r=\frac{1}{s\left|e^{i t}-e^{i u}\right|^{2-s}},
$$

and for $n \geq 1$

$$
\int_{R_{n}} \frac{r\left(1-r^{2}\right)^{s-1}}{\left|e^{i t}-r e^{i u}\right|^{2}} d r \leq \int_{R_{n}} \frac{d r}{(1-r)^{3-s}} \leq \frac{4 \cdot 2^{-n}}{\left|e^{i t}-e^{i u}\right|^{2-s}} .
$$

Together (4.5) and (4.6) gives

$$
\begin{aligned}
\int_{0}^{1} \frac{r\left(1-r^{2}\right)^{s-1}}{\left|e^{i t}-r e^{i u}\right|^{2}} d r & \leq \int_{R_{0}} \frac{r\left(1-r^{2}\right)^{s-1}}{\left|e^{i t}-r e^{i u}\right|^{2}} d r+\sum_{n=1}^{N} \int_{R_{n}} \frac{\left(1-r^{2}\right)^{s-1}}{\left|e^{i t}-r e^{i u}\right|^{2}} d r \\
& \leq \frac{1}{s\left|e^{i t}-e^{i u}\right|^{2-s}}+\frac{4}{\left|e^{i t}-e^{i u}\right|^{2-s}} \sum_{n=1}^{N} 2^{-n} \\
& \leq \frac{C}{\left|e^{i t}-e^{i u}\right|^{2-s}},
\end{aligned}
$$

proving (4.4). So, the proof is finished.

As a consequence of Proposition 4.2 we obtain the following result.

Corollary 4.3. Let $1<p<\infty, 0<s<1$, and let $f \in H^{1}$. Then $f \in B_{p}(s)$ if and only if $f_{b} \in L_{s}^{p}$, where $f_{b}$ denotes the boundary values of $f$ (taken as a radial limit or a nontangential limit). Moreover, $\|f\|_{B_{p}(s)} \asymp\|f\|_{L_{s}^{p} \text {. }}$

Proof. Since $f \in H^{1}$, then $f=\widehat{f}_{b}$ (see [13, Chapter II]). Hence the result follows from Proposition 4.2.

Multipliers of $L_{s}^{p}$. Let $M\left(L_{s}^{p}\right)$ be the algebra of (pointwise) multipliers of $L_{s}^{p}$, that is,

$$
M\left(L_{s}^{p}\right)=\left\{g: \mathbb{T} \rightarrow \mathbb{C}: g f \in L_{s}^{p} \text { whenever } f \in L_{s}^{p}\right\} .
$$

Note that, since $1 \in L_{s}^{p}$, we have $M\left(L_{s}^{p}\right) \subset L_{\alpha}^{p}$. We also need a description of the multipliers of $L_{s}^{p}$, in terms of Carleson measures for $B_{p}(s)$, in a similar way as Lemma F. To do this, first we need the following description of Carleson measures for $B_{p}(s)$ using functions in $L_{s}^{p}$.

Lemma 4.4. Let $1<p<\infty, 0<s<1$, and let $\mu$ be a positive measure on $\mathbb{D}$. The following conditions are equivalent:

(i) $\mu$ is an $(s, p)$-Carleson measure;

(ii) $\int_{\mathbb{D}}|\widehat{h}(z)|^{p} d \mu(z) \leq C\|h\|_{L_{s}^{p}}^{p}$ for all $h \in L_{s}^{p}$.

Proof. Suppose first that $\mu$ is an $(s, p)$-Carleson measure and let $h \in L_{s}^{p}$. Without loss of generality we may assume that $h$ is real valued. Let $\widetilde{h}$ be the harmonic conjugate function of $\widehat{h}$. By the Cauchy-Riemann equations we have $|\nabla \widehat{h}(z)| \asymp$ $\left|f^{\prime}(z)\right|$, where $f=\widehat{h}+i \widetilde{h}$. Now, it follows from Proposition 4.2 that $f$ belongs to $B_{p}(s)$. Then, by $(i)$ and (4.2),

$$
\begin{aligned}
\int_{\mathbb{D}}|\widehat{h}(z)|^{p} d \mu(z) & \lesssim \int_{\mathbb{D}}|f(z)|^{p} d \mu(z) \lesssim\|f\|_{B_{p}(s)}^{p} \\
& \lesssim\|h\|_{L^{p}(\mathbb{T})}^{p}+\int_{\mathbb{D}}|\nabla \widehat{h}(z)|^{p} d A_{p, s}(z) \lesssim\|h\|_{L_{s}^{p}}^{p} .
\end{aligned}
$$


For the converse, suppose that (ii) holds, and let $f \in B_{p}(s)$. By Corollary 4.3, $f_{b} \in L_{s}^{p}$ and $f=\widehat{f}_{b}$. So, by condition $(i i)$ and (4.1), we get

$$
\int_{\mathbb{D}}|f(z)|^{p} d \mu(z)=\int_{\mathbb{D}}\left|\widehat{f}_{b}(z)\right| d \mu(z) \leq C\left\|f_{b}\right\|_{L_{s}^{p}}^{p} \leq C\|f\|_{B_{p}(s)}^{p},
$$

and therefore $\mu$ is a Carleson measure for $B_{p}(s)$.

Lemma 4.5. Let $1<p<\infty, 0<s<1$. Let $f \in L^{\infty}(\mathbb{T})$ and $F \in C^{1}(\mathbb{D}) \cap L^{\infty}(\mathbb{D})$ with $\lim _{r \rightarrow 1} F\left(r e^{i \theta}\right)=f\left(e^{i \theta}\right)$ for a.e. $e^{i \theta} \in \mathbb{T}$. If $|\nabla F(z)|^{p} d A_{p, s}(z)$ is an $(s, p)$ Carleson measure, then $f \in M\left(L_{s}^{p}\right)$.

Proof. We must show that $f g \in L_{s}^{p}$ whenever $g$ is in $L_{s}^{p}$. So, let $g \in L_{s}^{p}$. Since $f \in L^{\infty}(\mathbb{T})$, it follows that $f g \in L^{p}(\mathbb{T})$. Using (4.1) with the extension $F \widehat{g}$ of $f g$ on $\mathbb{D}$ we get

$$
\begin{aligned}
\|f g\|_{L_{s}^{p}}^{* p} & \leq C \int_{\mathbb{D}}|\nabla(F \widehat{g})(z)|^{p} d A_{p, s}(z) \\
& \leq C\left(\int_{\mathbb{D}}|F(z)|^{p}|\nabla \widehat{g}(z)|^{p} d A_{p, s}(z)+\int_{\mathbb{D}}|\widehat{g}(z)|^{p}|\nabla F(z)|^{p} d A_{p, s}(z)\right) .
\end{aligned}
$$

Since $F \in L^{\infty}(\mathbb{D})$, for the first term of the sum, using (4.2) with $g \in L_{s}^{p}$ we obtain

$$
\int_{\mathbb{D}}|F(z)|^{p}|\nabla \widehat{g}(z)|^{p} d A_{p, s}(z) \leq C\|F\|_{L^{\infty}(\mathbb{D})}^{p}\|g\|_{L_{s}^{p}}^{p}
$$

For the second term, we use the fact that $|\nabla F(z)|^{p} d A_{p, s}(z)$ is an $(s, p)$-Carleson measure and Lemma 4.4 to get

$$
\int_{\mathbb{D}}|\widehat{g}(z)|^{p}|\nabla F(z)|^{p} d A_{p, s}(z) \leq C\|g\|_{L_{s}^{p}}^{p}
$$

Putting together (4.7), (4.8) and (4.9), we obtain that $\|f g\|_{L_{s}^{p}} \leq C\|g\|_{L_{s}^{p}}$. So $f \in M\left(L_{s}^{p}\right)$ and the proof is complete.

Now we can give a description of the multipliers of $L_{s}^{p}$ in terms of $(s, p)$-Carleson measures.

Theorem 4.6. Let $1<p<\infty$ and $0<s<1$. The following conditions are equivalent:

(i) $g \in M\left(L_{s}^{p}\right)$

(ii) $g \in L^{\infty}(\mathbb{T})$ and $|\nabla \widehat{g}(z)|^{p} d A_{p, s}(z)$ is an $(s, p)$-Carleson measure.

Proof. That $(i i) \Rightarrow(i)$ follows from Lemma 4.5. For the converse, let $g \in M\left(L_{s}^{p}\right)$. We start by proving that $g \in L^{\infty}(\mathbb{T})$ using an argument from [10]. By the closed graph theorem, the operator of multiplication by $g$ is bounded, that is, $\|g f\|_{L_{s}^{p}}^{p} \leq$ $C\|f\|_{L_{s}^{p}}^{p}$ for all $f \in L_{s}^{p}$. Taking $\varphi(z)=g(z) / C^{1 / p}$ we may assume that $\|\varphi f\|_{L_{s}^{p}}^{p} \leq$ $\|f\|_{L_{s}^{p}}^{p}$ for all $f \in L_{s}^{p}$. Note that

$$
\varphi^{n}\left(e^{i t}\right)\left(f\left(e^{i t}\right)-f\left(e^{i u}\right)\right)=\left(\varphi^{n} f\right)\left(e^{i t}\right)-\left(\varphi^{n} f\right)\left(e^{i u}\right)-f\left(e^{i u}\right)\left(\varphi^{n}\left(e^{i t}\right)-\varphi^{n}\left(e^{i u}\right)\right) .
$$


Since $1 \in L_{s}^{p}$, we see that $\varphi \in L_{s}^{p}$, and so $\varphi^{n} \in L_{s}^{p}$ with $\left\|\varphi^{n}\right\|_{L_{s}^{p}} \leq\|\varphi\|_{L_{s}^{p}}$, for $n \geq 1$. Hence,

$$
\begin{aligned}
\int_{0}^{2 \pi} \int_{0}^{2 \pi}\left|\varphi\left(e^{i t}\right)\right|^{n p} & \frac{\left|f\left(e^{i t}\right)-f\left(e^{i u}\right)\right|^{p}}{\left|e^{i t}-e^{i u}\right|^{2-s}} d t d u \\
& \leq C\left(\left\|\varphi^{n} f\right\|_{L_{s}^{p}}^{p}+\int_{0}^{2 \pi} \int_{0}^{2 \pi}\left|f\left(e^{i u}\right)\right|^{p} \frac{\left|\varphi^{n}\left(e^{i t}\right)-\varphi^{n}\left(e^{i u}\right)\right|^{p}}{\left|e^{i t}-e^{i u}\right|^{2-s}} d t d u\right) \\
& \leq C\left(\|f\|_{L_{s}^{p}}^{p}+\int_{0}^{2 \pi} \int_{0}^{2 \pi}\left|f\left(e^{i u}\right)\right|^{p} \frac{\left|\varphi^{n}\left(e^{i t}\right)-\varphi^{n}\left(e^{i u}\right)\right|^{p}}{\left|e^{i t}-e^{i u}\right|^{2-s}} d t d u\right)
\end{aligned}
$$

Now, taking $f$ to be the identity function on $\mathbb{T}$, which belongs to $L_{s}^{p}$, we obtain

$$
\begin{aligned}
\int_{0}^{2 \pi} & \int_{0}^{2 \pi}\left|\varphi\left(e^{i t}\right)\right|^{n p}\left|e^{i t}-e^{i u}\right|^{p-2+s} d t d u \\
& \leq C+C\left(\int_{0}^{2 \pi} \int_{0}^{2 \pi} \frac{\left|\varphi^{n}\left(e^{i t}\right)-\varphi^{n}\left(e^{i u}\right)\right|^{p}}{\left|e^{i t}-e^{i u}\right|^{2-s}} d t d u\right) \leq C .
\end{aligned}
$$

Therefore, since $n$ is arbitrary and the constant $C$ is independent of $n$, we have $|\varphi(\xi)| \leq C$ for almost every $\xi \in \mathbb{T}$ and so $g \in L^{\infty}(\mathbb{T})$.

Now we are going to show that the measure $|\nabla \widehat{g}(z)|^{p} d A_{p, s}(z)$ is an $(s, p)$-Carleson measure. So, let $f \in B_{p}(s)$. Using (4.2), (4.1) and the fact that $g \in M\left(L_{s}^{p}\right)$ we see that

$$
\int_{\mathbb{D}}|\nabla(f \widehat{g})(z)|^{p} d A_{p, s}(z) \leq C\|f g\|_{L_{s}^{p}} \leq C\|f\|_{L_{s}^{p}}^{p} \leq C\|f\|_{B_{p}(s)}^{p} .
$$

Furthermore, since $g \in L^{\infty}(\mathbb{T})$ then $\widehat{g} \in L^{\infty}(\mathbb{D})$ and we obtain

$$
\int_{\mathbb{D}}|\widehat{g}(z)|^{p}|\nabla f(z)|^{p} d A_{p, s}(z) \leq C\|f\|_{B_{p}(s)}^{p} .
$$

Finally, putting together (4.10) and (4.11), we get

$$
\begin{aligned}
\int_{\mathbb{D}}|f(z)|^{p}|\nabla \widehat{g}(z)|^{p} d A_{p, s}(z) & \leq C \int_{\mathbb{D}}\left(|\nabla(f \widehat{g})(z)|^{p}+|\widehat{g}(z)|^{p}|\nabla f(z)|^{p}\right) d A_{p, s}(z) \\
& \leq C\|f\|_{B_{p}(s)}^{p} .
\end{aligned}
$$

Therefore, the measure $|\nabla \widehat{g}(z)|^{p} d A_{p, s}(z)$ is an $(s, p)$-Carleson measure and the proof is complete.

As a consequence of Theorem 4.6 we can conclude the following relationship between multipliers in $B_{p}(s)$ and multipliers in $L_{s}^{p}$.

Corollary 4.7. Let $f \in H^{1}$. Then $f \in M\left(B_{p}(s)\right)$ if and only if $f_{b} \in M\left(L_{s}^{p}\right)$.

Proof. Let $f$ be analytic on $\mathbb{D}$. By Lemma $\mathrm{F}, f \in M\left(B_{p}(s)\right)$ if and only if $f \in H^{\infty}$ and $\left|f^{\prime}(z)\right|^{p} d A_{p, s}(z)$ is an $(s, p)$-Carleson measure. Since $f \in H^{1}$, then $\widehat{f}_{b}=f$. So, by Theorem 4.6, $f \in M\left(B_{p}(s)\right)$ if and only if $f_{b} \in M\left(L_{s}^{p}\right)$. 


\section{NeCEssity of CONDItion (CS)}

Recall that for $s>0$, the $Q_{s}$ space consists of those analytic functions $f$ on $\mathbb{D}$ for which the measure

$$
d \mu_{f, s}(z)=\left|f^{\prime}(z)\right|^{2}\left(1-|z|^{2}\right)^{s} d A(z)
$$

is an $s$-Carleson measure, that is, there is a positive constant $C$ such that for any Carleson box $S(I)$,

$$
\mu_{f, s}(S(I)) \leq C|I|^{s} .
$$

A sequence of points $\left\{z_{n}\right\} \subset \mathbb{D}$ is called an interpolating sequence for $Q_{s} \cap H^{\infty}$ if for any bounded sequence $\left\{a_{n}\right\}$ of complex numbers, we can find a function $f \in Q_{s} \cap H^{\infty}$ with $f\left(z_{n}\right)=a_{n}$ for each $n$. In [21] the following characterization of interpolating sequences for $Q_{s} \cap H^{\infty}$ was obtained.

Theorem G. Let $0<s<1$, and let $\left\{z_{n}\right\} \subset \mathbb{D}$. Then $\left\{z_{n}\right\}$ is an interpolating sequence for $Q_{s} \cap H^{\infty}$ if and only if the sequence $\left\{z_{n}\right\}$ is separated and

$$
\sum_{n}\left(1-\left|z_{n}\right|^{2}\right)^{s} \delta_{z_{n}}
$$

is an $\mathrm{s}$-Carleson measure.

Lemma 5.1. Let $1<p \leq 2$, and $0<s<1$. Then $M\left(B_{p}(s)\right) \subset Q_{s} \cap H^{\infty}$.

Proof. Let $f \in M\left(B_{p}(s)\right)$. By Lemma F, $f \in H^{\infty}$ and the measure $d \mu_{f, p, s}(z)=$ $\left|f^{\prime}(z)\right|^{p} d A_{p, s}(z)$ is an $(s, p)$-Carleson measure. By Lemma 3.1, this implies that the measure $\mu_{f, p, \alpha}$ is an $s$-Carleson measure. Also, since $H^{\infty} \subset \mathcal{B}$, we have $(1-$ $\left.|z|^{2}\right)\left|f^{\prime}(z)\right| \leq C$. Therefore, since $p \leq 2$, for any Carleson box $S(I)$, we get

$$
\begin{aligned}
\int_{S(I)}\left|f^{\prime}(z)\right|^{2}\left(1-|z|^{2}\right)^{s} d A(z) & =\int_{S(I)}\left|f^{\prime}(z)\right|^{p}\left|f^{\prime}(z)\right|^{2-p}\left(1-|z|^{2}\right)^{s} d A(z) \\
& \leq C \int_{S(I)}\left|f^{\prime}(z)\right|^{p}\left(1-|z|^{2}\right)^{p-2+s} d A(z) \\
& \leq C|I|^{s}
\end{aligned}
$$

Hence the measure $\left|f^{\prime}(z)\right|^{2}\left(1-|z|^{2}\right)^{s} d A(z)$ is an $s$-Carleson measure, and so $f$ belongs to $Q_{s}$.

Lemma 5.2. Let $1<p \leq 2,0<s<1$. If $\left\{z_{j}\right\}$ is an interpolating sequence for $M\left(B_{p}(s)\right)$, then

$$
\sup _{z \in \mathbb{D}} \sum_{k=1}^{\infty} \frac{\left(1-|z|^{2}\right)^{s}\left(1-\left|z_{k}\right|^{2}\right)^{s}}{\left|1-\bar{z}_{k} z\right|^{2 s}}<\infty .
$$

Proof. This follows directly from Lemma 5.1, Theorem G and Lemma D.

$(\mathbf{M}) \Rightarrow(\mathbf{C S})$. Suppose that $(\mathrm{M})$ holds. Since $\left\{z_{j}\right\}$ is an interpolating sequence for $M\left(B_{p}(s)\right) \subset H^{\infty}$, in particular $\left\{z_{j}\right\}$ is an interpolating sequence for $H^{\infty}$, hence, it is separated.

The Carleson measure condition will follow from an argument which combines Khinchine's inequality and the reproducing formula for $B_{p}(s)$. The idea to use Khinchine's inequality on interpolation problems goes back to Varopoulos (see [28]). 
Fix a positive integer $n>1$, and set $\varepsilon_{j}^{k}= \pm 1$ for $1 \leq k \leq n$ and $1 \leq j \leq 2^{n}$. Take $g_{j} \in M\left(B_{p}(s)\right)$ with $g_{j}\left(z_{k}\right)=\varepsilon_{j}^{k}$, and let $f \in B_{p}(s)$. Since $f g_{j} \in B_{p}(s)$ with $\left\|f g_{j}\right\|_{B_{p}(s)} \leq C\|f\|_{B_{p}(s)}$, using the reproducing formula (1.1), we get

$$
\left(f g_{j}\right)\left(z_{k}\right)=\left(f g_{j}\right)(0)+\int_{\mathbb{D}}\left(f g_{j}\right)^{\prime}(w) K\left(z_{k}, w\right)\left(1-|w|^{2}\right)^{s} d A(w),
$$

where

$$
K\left(z_{k}, w\right)=\frac{\left(1-\bar{w} z_{k}\right)^{1+s}-1}{\bar{w}\left(1-\bar{w} z_{k}\right)^{1+s}}
$$

In particular, we have the estimate

$$
\left|K\left(z_{k}, w\right)\right| \lesssim \frac{1}{\left|1-\bar{w} z_{k}\right|^{1+s}}
$$

Now, by (5.2), we have

$$
\begin{aligned}
& \sum_{k=1}^{n}\left|f\left(z_{k}\right)\right|^{p}\left(1-\left|z_{k}\right|^{2}\right)^{s}=\sum_{k=1}^{n} \varepsilon_{k}^{j}\left(f g_{j}\right)\left(z_{k}\right) \overline{f\left(z_{k}\right)}\left|f\left(z_{k}\right)\right|^{p-2}\left(1-\left|z_{k}\right|^{2}\right)^{s} \\
& \quad=\left(f g_{j}\right)(0) \sum_{k=1}^{n} \varepsilon_{k}^{j} \overline{f\left(z_{k}\right)}\left|f\left(z_{k}\right)\right|^{p-2}\left(1-\left|z_{k}\right|^{2}\right)^{s} \\
& \quad+\sum_{k=1}^{n} \varepsilon_{k}^{j}\left(\int_{\mathbb{D}}\left(f g_{j}\right)^{\prime}(w) K\left(z_{k}, w\right)\left(1-|w|^{2}\right)^{s} d A(w)\right) \overline{f\left(z_{k}\right)}\left|f\left(z_{k}\right)\right|^{p-2}\left(1-\left|z_{k}\right|^{2}\right)^{s} \\
& \quad=I_{1}+I_{2} .
\end{aligned}
$$

We will compute the expectation of both sides of this equality. Let $q$ be the conjugate exponent of $p$. Since $|g(0)| \leq\|g\|_{B_{p}(s)}$ for all $g \in B_{p}(s)$, applying Khinchine's inequality (2.3) with $r=1$,

$$
\begin{aligned}
\mathcal{E}\left(\left|I_{1}\right|\right) & \leq\left\|f g_{j}\right\|_{B_{p}(s)} \mathcal{E}\left(\left.\left|\sum_{k=1}^{n} \varepsilon_{k}^{j} \overline{f\left(z_{k}\right)}\right| f\left(z_{k}\right)\right|^{p-2}\left(1-\left|z_{k}\right|^{2}\right)^{s} \mid\right) \\
& \leq C\|f\|_{B_{p}(s)}\left(\sum_{k=1}^{n}\left|f\left(z_{k}\right)\right|^{2(p-1)}\left(1-\left|z_{k}\right|^{2}\right)^{2 s}\right)^{1 / 2} .
\end{aligned}
$$

- If $p \geq 2$, then $2(p-1) \geq p$, and by (2.5) with $r=\frac{p}{2(p-1)}=q / 2 \leq 1$ we get

$$
\begin{aligned}
\mathcal{E}\left(\left|I_{1}\right|\right) & \leq C\|f\|_{B_{p}(s)}\left(\sum_{k=1}^{n}\left|f\left(z_{k}\right)\right|^{p}\left(1-\left|z_{k}\right|^{2}\right)^{q s}\right)^{1 / q} \\
& \leq C\|f\|_{B_{p}(s)}\left(\sum_{k=1}^{n}\left|f\left(z_{k}\right)\right|^{p}\left(1-\left|z_{k}\right|^{2}\right)^{s}\right)^{1 / q} .
\end{aligned}
$$

- If $1<p<2$ we apply Holder's inequality with exponent $q / 2 \geq 1$, and then (5.1) with $z=0$, to get

$$
\begin{aligned}
\mathcal{E}\left(\left|I_{1}\right|\right) & \lesssim\|f\|_{B_{p}(s)}\left(\sum_{k=1}^{n}\left|f\left(z_{k}\right)\right|^{q(p-1)}\left(1-\left|z_{k}\right|^{2}\right)^{2 s}\right)^{1 / q}\left(\sum_{k=1}^{n}\left(1-\left|z_{k}\right|^{2}\right)^{2 s}\right)^{1 / p} \\
& \lesssim\|f\|_{B_{p}(s)}\left(\sum_{k=1}^{n}\left|f\left(z_{k}\right)\right|^{p}\left(1-\left|z_{k}\right|^{2}\right)^{s}\right)^{1 / q}
\end{aligned}
$$


Together (5.4) and (5.5), gives that there is a constant $C$ independent of $n$ such that

$$
\mathcal{E}\left(\left|I_{1}\right|\right) \leq C\|f\|_{B_{p}(s)}\left(\sum_{k=1}^{n}\left|f\left(z_{k}\right)\right|^{p}\left(1-\left|z_{k}\right|^{2}\right)^{s}\right)^{1 / q}, \quad 1<p<\infty .
$$

Now, we are going to estimate the expectation of $I_{2}$. We have

$$
\begin{aligned}
\mathcal{E}\left(\left|I_{2}\right|\right) & \leq\left\|f g_{j}\right\|_{B_{p}(s)} \\
& \left(\int_{\mathbb{D}}\left(\left.\mathcal{E}\left|\sum_{k=1}^{n} \varepsilon_{k}^{j} K\left(z_{k}, w\right) \overline{f\left(z_{k}\right)}\right| f\left(z_{k}\right)\right|^{p-2}\left(1-\left|z_{k}\right|^{2}\right)^{s} \mid\right)^{q} d A_{q, s}(w)\right)^{1 / q}
\end{aligned}
$$

(by Hölder's inequality)

$$
\begin{aligned}
& \lesssim\|f\|_{B_{p}(s)} \\
& \left(\int_{\mathbb{D}} \mathcal{E}\left(\left.\left.\left|\sum_{k=1}^{n} \varepsilon_{k}^{j} K\left(z_{k}, w\right) \overline{f\left(z_{k}\right)}\right| f\left(z_{k}\right)\right|^{p-2}\left(1-\left|z_{k}\right|^{2}\right)^{s}\right|^{q}\right) d A_{q, s}(w)\right)^{1 / q}
\end{aligned}
$$

(by (2.4))

$\lesssim\|f\|_{B_{p}(s)}\left(\int_{\mathbb{D}}\left(\sum_{k=1}^{n}\left|K\left(z_{k}, w\right)\right|^{2}\left|f\left(z_{k}\right)\right|^{2(p-1)}\left(1-\left|z_{k}\right|^{2}\right)^{2 s}\right)^{q / 2} d A_{q, s}(w)\right)^{1 / q}$

(by (2.3) with $r=q$ ).

- If $p \geq 2$, we use inequality (2.5) with $r=q / 2 \leq 1$ in the last estimate to obtain

$$
\begin{aligned}
\mathcal{E}\left(\left|I_{2}\right|\right) & \lesssim\|f\|_{B_{p}(s)} \\
& \left(\int_{\mathbb{D}}\left(\sum_{k=1}^{n}\left|K\left(z_{k}, w\right)\right|^{q} \cdot\left|f\left(z_{k}\right)\right|^{q(p-1)}\left(1-\left|z_{k}\right|^{2}\right)^{q s}\right) d A_{q, s}(w)\right)^{1 / q} \\
& \lesssim\|f\|_{B_{p}(s)}\left(\sum_{k=1}^{n}\left|f\left(z_{k}\right)\right|^{p}\left(1-\left|z_{k}\right|^{2}\right)^{q s} \int_{\mathbb{D}} \frac{\left(1-|w|^{2}\right)^{q-2+s}}{\left|1-\bar{w} z_{k}\right|^{(1+s) q}} d A(w)\right)^{1 / q} \\
& \lesssim\|f\|_{B_{p}(s)}\left(\sum_{k=1}^{n}\left|f\left(z_{k}\right)\right|^{p}\left(1-\left|z_{k}\right|^{2}\right)^{s}\right)^{1 / q}
\end{aligned}
$$

after and application of Lemma A (we can apply it, since $(1+s) q>2+(q-2+s)$ ).

- If $1<p<2$, we use Hölder's inequality with exponent $q / 2 \geq 1$, and then 
(5.1) to obtain

$$
\begin{aligned}
& \mathcal{E}\left(\left|I_{2}\right|\right) \lesssim\|f\|_{B_{p}(s)} \\
& \left(\int_{\mathbb{D}}\left(\sum_{k=1}^{n}\left|f\left(z_{k}\right)\right|^{p} \frac{\left(1-\left|z_{k}\right|^{2}\right)^{s+\frac{q s}{2}}}{|1-\bar{w} z|^{q+2 s}}\right)\left(\sum_{k=1}^{n} \frac{\left(1-\left|z_{k}\right|^{2}\right)^{s}}{\left|1-\bar{w} z_{k}\right|^{2 s}}\right)^{(q-2) / 2} d A_{q, s}(w)\right)^{1 / q} \\
& \lesssim\|f\|_{B_{p}(s)}\left(\int_{\mathbb{D}}\left(\sum_{k=1}^{n}\left|f\left(z_{k}\right)\right|^{p} \frac{\left(1-\left|z_{k}\right|^{2}\right)^{s+\frac{q s}{2}}}{|1-\bar{w} z|^{q+2 s}}\right)\left(1-|w|^{2}\right)^{-s(q-2) / 2} d A_{q, s}(w)\right)^{1 / q} \\
& \quad=\|f\|_{B_{p}(s)}\left(\sum_{k=1}^{n}\left|f\left(z_{k}\right)\right|^{p}\left(1-\left|z_{k}\right|^{2}\right)^{s+\frac{q s}{2}} \int_{\mathbb{D}} \frac{\left(1-|w|^{2}\right)^{q+2 s-2-s \frac{q}{2}}}{|1-\bar{w} z|^{q+2 s}} d A(w)\right)^{1 / q} \\
& \quad \lesssim\|f\|_{B_{p}(s)}\left(\sum_{k=1}^{n}\left|f\left(z_{k}\right)\right|^{p}\left(1-\left|z_{k}\right|^{2}\right)^{s}\right)^{1 / q},
\end{aligned}
$$

after an application of Lemma A. Putting together the estimates for $\mathcal{E}\left(\left|I_{2}\right|\right)$ obtained for the cases $1<p<2$ and $p \geq 2$, we have that there is a constant $C$ independent of $n$ such that

$$
\mathcal{E}\left(\left|I_{2}\right|\right) \leq C\|f\|_{B_{p}(s)}\left(\sum_{k=1}^{n}\left|f\left(z_{k}\right)\right|^{p}\left(1-\left|z_{k}\right|^{2}\right)^{s}\right)^{1 / q}
$$

Then, together (5.6) and (5.7) gives

$$
\sum_{k=1}^{n}\left|f\left(z_{k}\right)\right|^{p}\left(1-\left|z_{k}\right|^{2}\right)^{s} \leq \mathcal{E}\left(\left|I_{1}\right|\right)+\mathcal{E}\left(\left|I_{2}\right|\right) \leq C\|f\|_{B_{p}(s)}\left(\sum_{k=1}^{n}\left|f\left(z_{k}\right)\right|^{p}\left(1-\left|z_{k}\right|^{2}\right)^{s}\right)^{1 / q},
$$

where the constant $C$ is independent of $n$. So, we can conclude that

$$
\sum_{k=1}^{\infty}\left|f\left(z_{k}\right)\right|^{p}\left(1-\left|z_{k}\right|^{2}\right)^{s} \leq C\|f\|_{B_{p}(s)}^{p}
$$

that is, the measure $\sum_{z_{k} \in Z}\left(1-\left|z_{k}\right|^{2}\right)^{s} \delta_{z_{k}}$ is a Carleson measure for $B_{p}(s)$. This finishes the proof.

(UIS) $\Rightarrow$ (CS). Let $Z=\left\{z_{n}\right\}$, and suppose the map $T_{Z}: f \mapsto\left\{\frac{f\left(z_{n}\right)}{\left\|k_{n}\right\|_{B_{q}(s)}}\right\}$ from $B_{p}(s)$ to $\ell^{p}$, is bounded and onto. The Carleson measure condition is just a reformulation of the boundedness of the map. Indeed, let $f \in B_{p}(s)$, then

$$
\begin{aligned}
\int_{\mathbb{D}}|f(z)|^{p} d \mu_{Z}(z) & =\sum\left|f\left(z_{n}\right)\right|^{p}\left(1-\left|z_{n}\right|^{2}\right)^{s} \\
& \leq C \sum \frac{\left|f\left(z_{n}\right)\right|^{p}}{\left\|k_{n}\right\|_{B_{q}(s)}^{p}}=C\left\|T_{Z} f\right\|_{\ell^{p}}^{p} \leq C\|f\|_{B_{p}(s)}^{p} .
\end{aligned}
$$

Now we prove the separation condition. Fix a point $z_{j} \in Z$. Since $T_{Z}$ is onto, there exists a function $f_{j} \in B_{p}(s)$ such that $f_{j}\left(z_{j}\right)=1$, and $f_{j}\left(z_{k}\right)=0$ if $k \neq j$. Now, it follows from the open mapping theorem and Lemma 2.2 that

$$
\left\|f_{j}\right\|_{B_{p}(s)}^{p} \leq C \sum_{k} \frac{\left|f\left(z_{k}\right)\right|^{p}}{\left\|k_{z_{k}}\right\|_{B_{q}(s)}^{p}} \leq C\left|f\left(z_{j}\right)\right|^{p}\left(1-\left|z_{j}\right|^{2}\right)^{s} .
$$


Now, let $z_{m}$ be a point in $Z$ distinct from $z_{j}$. We can assume that $\rho\left(z_{j}, z_{m}\right) \leq 1 / 2$. Then, it follows from (5.8) and Lemma 2.3 that

$$
\begin{aligned}
\left\|f_{j}\right\|_{B_{p}(s)} & \leq C\left|f\left(z_{j}\right)-f\left(z_{m}\right)\right|\left(1-\left|z_{j}\right|^{2}\right)^{s / p} \\
& \leq C\left\|f_{j}\right\|_{B_{p}(s)}\left\|k_{z_{j}}-k_{z_{m}}\right\|_{B_{q}(s)}\left(1-\left|z_{j}\right|^{2}\right)^{s / p} \\
& \leq C\left\|f_{j}\right\|_{B_{p}(s)} \rho\left(z_{j}, z_{m}\right) .
\end{aligned}
$$

Hence $\rho\left(z_{j}, z_{m}\right) \geq \delta$ with $\delta=1 / C$, and the sequence $\left\{z_{n}\right\}$ is separated.

\section{A $\bar{\partial}$-PROBlem With ESTIMATES AND THE CORONA THEOREM}

In order to prove the sufficiency of condition $(C S)$ in Theorem 1, first we will construct a non-analytic solution of the interpolation problem, and then we find the analytic solution by solving a $\bar{\partial}$-equation with the appropriate estimates.

Lemma 6.1. Let $1<p<\infty$ and $0 \leq s<1$. Then $e^{-i \theta} \in M\left(L_{s}^{p}\right)$.

Proof. Note that $h \in M\left(L_{s}^{p}\right)$ if and only if $\bar{h} \in M\left(L_{s}^{p}\right)$. So, it is enough to show that $h\left(e^{i \theta}\right)=e^{i \theta} \in M\left(L_{s}^{p}\right)$. Let $\widehat{h}(z)=z$ be the harmonic extension of $h$ in $\mathbb{D}$. By Theorem 4.6, it is enough to show that $d A_{p, s}(z)=\left(1-|z|^{2}\right)^{p-2+s} d A(z)$ is an $(s, p)$-Carleson measure. So, let $f \in B_{p}(s)$. Using Lemma B with $b=0$ and $\sigma=p-2+s$, we get

$$
\begin{aligned}
\int_{\mathbb{D}}|f(z)|^{p} d A_{p, s}(z) & \leq C|f(0)|^{p}+C \int_{\mathbb{D}}|f(z)-f(0)|^{p}\left(1-|z|^{2}\right)^{p-2+s} d A(z) \\
& \leq C|f(0)|^{p}+C \int_{\mathbb{D}}\left|f^{\prime}(z)\right|^{p}\left(1-|z|^{2}\right)^{2 p-2+s} d A(z) \\
& \leq C\|f\|_{B_{p}(s)}^{p} .
\end{aligned}
$$

Hence the measure $\left(1-|z|^{2}\right)^{p-2+s} d A(z)$ is an $(s, p)$-Carleson measure and the proof is complete.

First of all we will solve a $\bar{\partial}$-equation that will be useful in the construction of the interpolating function $f \in B_{p}(s)$

Theorem 6.2. Let $1<p<\infty$ and $0<s<1$. If $\phi \in \mathcal{C}(\overline{\mathbb{D}})$, then there is a function $b \in \mathcal{C}(\overline{\mathbb{D}})$ such that

$$
\frac{\partial b}{\partial \bar{z}}=\phi \quad \text { on } \mathbb{D},
$$

(in the sense of distributions) such that the boundary valued function $b_{\mid \mathbb{T}}$ belongs to $L_{s}^{p}$. Moreover $\left\|b_{\mid \mathbb{T}}\right\|_{L_{s}^{p}} \lesssim\|\phi\|_{L^{p}\left(d A_{p, s}\right)}$.

Proof. Consider the function

$$
u(z)=\int_{\mathbb{D}} \frac{\phi(w)}{(z-w)} d A(w) .
$$

It is easy to check that $u \in \mathcal{C}(\overline{\mathbb{D}})$ and $\bar{\partial} u=\phi$ (see [13, Chapter VIII]). We will show that $u_{\mid \mathbb{T}}$ belongs to $L_{s}^{p}$. To do that, consider the function

$$
v(z)=\int_{\mathbb{D}} \frac{\phi(w)}{(1-w \bar{z})} d A(w) .
$$


Observe that $v\left(e^{i \theta}\right)=e^{i \theta} u\left(e^{i \theta}\right)$ for all $e^{i \theta} \in \mathbb{T}$. So, by Lemma 6.1 and Lemma 4.1, it is enough to prove that $|\nabla v| \in L^{p}\left(d A_{p, s}\right)$. An easy computation gives

If we define

$$
|\nabla v(z)| \lesssim \int_{\mathbb{D}} \frac{|g(w)|}{|1-\bar{w} z|^{2}} d A(w)
$$

$$
T \phi(z)=\int_{\mathbb{D}} \frac{\phi(w)}{|1-\bar{w} z|^{2}} d A(w)
$$

then $T$ is a bounded operator from $L^{p}\left(d A_{p, s}\right)$ to itself (see [14, Theorem 1.9]). Since $\phi \in L^{p}\left(d A_{p, s}\right)$,

$$
\||\nabla v|\|_{L^{p}\left(d A_{p, s}\right)} \lesssim\|(T|\phi|)\|_{L^{p}\left(d A_{p, s}\right)} \lesssim\|\phi\|_{L^{p}\left(d A_{p, s}\right)}<\infty .
$$

Hence $|\nabla v| \in L^{p}\left(d A_{p, s}\right)$ and the proof is complete.

To prove the implication $(C S) \Rightarrow(M)$ in Theorem 1 , we need to solve a $\bar{\partial}$ problem in a way that the boundary values of the solution must be in $M\left(L_{s}^{p}\right)$. This is more technical than Theorem 6.2, and uses the beautiful solution of the $\bar{\partial}$-equation given by P. Jones in [16]. So, we pause briefly to discuss Jones' solution. Given a 1-Carleson measure $\mu$ on the unit disc, it is well known (see [13, Chapter VIII]) that the $\bar{\partial}$-problem $\bar{\partial} F=\mu$, has a solution $u$, in the sense of distributions, satisfying $\|u\|_{L^{\infty}(\mathbb{T})} \leq C\|\mu\|_{1}$. In [16], P. Jones found that one such solution is given by the formula

$$
u(z)=\int_{\mathbb{D}} K_{\mu}(z, \zeta) d \mu(\zeta)
$$

where

$$
K_{\mu}(z, \zeta)=\frac{2 i}{\pi} \frac{1-|\zeta|^{2}}{(1-\bar{\zeta} z)(z-\zeta)} \exp \left\{\int_{|w| \geq|\zeta|}\left(\frac{1+\bar{w} \zeta}{1-\bar{w} \zeta}-\frac{1+\bar{w} z}{1-\bar{w} z}\right) \frac{d \mu(w)}{\|\mu\|_{1}}\right\}
$$

The estimate $\int_{\mathbb{D}}\left|K_{\mu}\left(e^{i \theta}, \zeta\right)\right| d \mu(\zeta) \leq C_{1}\|\mu\|_{1}$ shows that $u \in L^{\infty}(\mathbb{T})$. Therefore, if $|g(z)| d A(z)$ is a 1-Carleson measure, then the equation $\bar{\partial} u=g$ has a solution $u \in L^{\infty}(\mathbb{T})$.

Before stating the $\bar{\partial}$-criteria needed, we prove the following simple observation.

Lemma 6.3. Let $1<p<\infty, 0<s<1$. If $|g(z)|^{p} d A_{p, s}(z)$ is an $(s, p)$-Carleson measure, then $|g(z)| d A(z)$ is a 1-Carleson measure.

Proof. Let $S(I)$ be a Carleson box. By Hölder's inequality and Lemma 3.1,

$$
\begin{aligned}
& \int_{S(I)}|g(z)| d A(z)=\int_{S(I)}|g(z)|\left(1-|z|^{2}\right)^{\frac{p-2+s}{p}}\left(1-|z|^{2}\right)^{\frac{-(p-2+s)}{p}} d A(z) \\
& \leq\left(\int_{S(I)}|g(z)|^{p} d A_{p, s}(z)\right)^{\frac{1}{p}}\left(\int_{S(I)}\left(1-|z|^{2}\right)^{\frac{-q}{p}(p-2+s)} d A(z)\right)^{\frac{1}{q}} \\
& \leq C|I|^{s / p}\left(\int_{S(I)} \frac{d A(z)}{\left(1-|z|^{2}\right)^{\frac{p-2+s}{p-1}}}\right)^{\frac{p-1}{p}} \\
& \leq C|I|^{s / p}\left(|I|^{\frac{p-s}{p-1}}\right)^{\frac{p-1}{p}}=C|I|
\end{aligned}
$$


Note that, in the last inequality, we used the fact that $s<1$.

Theorem 6.4. Let $1<p<\infty$ and $0<s<1$. If $|g(z)|^{p} d A_{p, s}(z)$ is an $(s, p)$ Carleson measure (and $|g(z)|(1-|z|) \leq C$ for $1<p<2)$, then there is a function $f$ defined on $\overline{\mathbb{D}}$ such that

$$
\frac{\partial f}{\partial \bar{z}}=g \quad \text { on } \mathbb{D}
$$

(in the sense of distributions) and such that the boundary valued function $f_{\mid \mathbb{T}}$ belongs to $M\left(L_{s}^{p}\right)$.

Proof. Since $|g(z)|^{p} d A_{p, s}(z)$ is an $(s, p)$-Carleson measure, it follows from Lemma 6.3 that $d \mu(z)=|g(z)| d A(z)$ is a 1-Carleson measure. Thus, we can take Jones' solution $u$, given by $(6.1)$, of the $\bar{\partial}$-problem $\bar{\partial} u=g$ on $\mathbb{D}$. Moreover, the boundary valued function $u$ lies in $L^{\infty}(\mathbb{T})$. But our aim is to verify that the boundary valued function $u$ lies in $M\left(L_{s}^{p}\right)$. For this purpose, consider

$v(z)=\frac{2 i}{\pi} \int_{\mathbb{D}} \frac{1-|\xi|^{2}}{|1-\bar{\xi} z|^{2}} \cdot \exp \left[\int_{|w| \geq|\xi|}\left(\frac{1+\bar{w} \xi}{1-\bar{w} \xi}-\frac{1+\bar{w} z}{1-\bar{w} z}\right)|g(w)| d A(w)\right] g(\xi) d A(\xi)$

which has the same boundary values as $z f(z)$. Then, by Lemma 6.1 and Lemma 4.5 , to see that $u_{\mid \mathbb{T}} \in M\left(L_{s}^{p}\right)$, it is enough to prove that $v \in L^{\infty}(\mathbb{D})$ and the measure $|\nabla v(z)|^{p} d A_{p, s}(z)$ is an $(s, p)$-Carleson measure. Note that the function $v$ was also considered in $[21]$, where the estimate

$$
|\nabla v(z)| \leq C \int_{\mathbb{D}} \frac{|g(w)|}{|1-\bar{w} z|^{2}} d A(w)
$$

was proved. Since $|g(z)| d A_{p, s}(z)$ is an $(s, p)$-Carleson measure (and if $1<p<2$, we have $\left.\left(1-|z|^{2}\right)|g(z)| \leq C\right)$, it follows from (6.2) and Lemma E that the measure $|\nabla v(z)|^{p} d A_{p, s}(z)$ is an $(s, p)$-Carleson measure.

Also, since $|g(z)| d A(z)$ is a Carleson measure, it follows from Lemma D that

$$
\operatorname{Re}\left(\int_{|w| \geq|\zeta|} \frac{1+\bar{w} \zeta}{1-\bar{w} \zeta}|g(w)| d A(w)\right) \leq 2 \int_{\mathbb{D}} \frac{1-|\zeta|^{2}}{|1-\bar{w} \zeta|^{2}}|g(w)| d A(w) \leq C,
$$

where $C>0$ is a constant independent of $\zeta \in \mathbb{D}$. This gives

$$
|v(z)| \lesssim \int_{\mathbb{D}} \frac{1-|\zeta z|^{2}}{|1-\bar{\zeta} z|^{2}} \exp \left(-\int_{|w| \geq|\zeta|} \frac{1-|w z|^{2}}{|1-\bar{w} z|^{2}}|g(w)| d A(w)\right)|g(\zeta)| d A(\zeta) \lesssim 1
$$

(see the proof of Lemma 2.1 in $[16]$ ). This implies that $v \in L^{\infty}(\mathbb{D})$ finishing the proof.

The corona problem. It is well known that there is a close connection between interpolating sequences, the $\bar{\partial}$-equation and the corona theorem. Here we will use Theorem 6.4 to show that the corona theorem holds for the algebra $M\left(B_{p}(s)\right)$. This was first proved by Tolokonnikov in [27].

Theorem 6.5. Let $1<p<\infty$ and $0<s<1$. Let $g_{1}, \ldots, g_{n} \in M\left(B_{p}(s)\right)$ with

$$
\inf _{z \in \mathbb{D}} \sum_{j=1}^{n}\left|g_{j}(z)\right|:=\delta>0 \text {. }
$$

Then there exist functions $f_{1}, \ldots, f_{n} \in M\left(B_{p}(s)\right)$ such that

$$
f_{1} g_{1}+\cdots+f_{n} g_{n} \equiv 1 \text {. }
$$


Proof. Once we have the $\bar{\partial}$ criteria needed (in our case, Theorem 6.4 ), the proof is very standard. Let $g_{1}, \ldots, g_{n} \in M\left(B_{p}(s)\right)$ for which (6.3) holds. Consider the non analytic solutions

$$
\varphi_{j}=\frac{\overline{g_{j}}}{\sum\left|g_{k}\right|^{2}}, \quad j=1, \ldots, n
$$

of the equation $g_{1} \varphi_{1}+\cdots+g_{n} \varphi_{n}=1$. For $1 \leq j, k \leq n$, let $G_{j k}=\varphi_{j} \bar{\partial} \varphi_{k}$. Note that, by (6.3), we have

$$
\left|G_{j, k}(z)\right| \lesssim \delta^{-3} \sum_{l=1}^{n}\left|g_{l}^{\prime}(z)\right| .
$$

By Lemma $\mathrm{F}$ we have that, for $1 \leq l \leq n$, the measures $\left|g_{l}^{\prime}(z)\right|^{p} d A_{p, s}(z)$ are $(s, p)$ Carleson measures. Then, by (6.4), if $f \in B_{p}(s)$,

$$
\begin{aligned}
\int_{\mathbb{D}}|f(z)|^{p}\left|G_{j k}(z)\right|^{p} d A_{p, s}(z) & \lesssim C_{n} \sum_{l=1}^{n} \int_{\mathbb{D}}|f(z)|^{p}\left|g_{l}^{\prime}(z)\right|^{p} d A_{p, s}(z) \\
& \leq C_{n}\|f\|_{B_{p}(s)}^{p}
\end{aligned}
$$

and so, $\left|G_{j k}(z)\right|^{p} d A_{p, s}(z)$ is an $(s, p)$-Carleson measure. Hence, by Theorem 6.4, there exist functions $a_{j k}$ with boundary values in $M\left(L_{s}^{p}\right)$ such that $\bar{\partial} a_{j k}=G_{j k}$ for $1 \leq j, k \leq n$. Then the functions

$$
f_{j}=\varphi_{j}+\sum_{k=1}^{n}\left(a_{j k}-a_{k j}\right) g_{k}, \quad j=1, \ldots, n,
$$

are analytic solutions of the equation $\sum_{j=1}^{n} f_{j} g_{j}=1$. To see that $f_{j} \in B_{p}(s)$, by Corollary 4.7 , it is enough to show that $f_{j}$ has boundary values in $M\left(L_{s}^{p}\right)$. Since $g_{j} \in M\left(B_{p}(s)\right)$ and $a_{j k}$ has boundary values in $M\left(L_{s}^{p}\right)$, all reduces to prove that the boundary values of $\varphi_{j}$ are in $M\left(L_{s}^{p}\right)$. To see that, by Lemma 4.5, it suffices to prove that $\varphi_{j}$ is bounded on $\mathbb{D}$, and $\left|\nabla \varphi_{j}(z)\right|^{p} d A_{p, s}$ is an $(s, p)$-Carleson measure. But, since $g_{j} \in M\left(B_{p}(s)\right) \subset H^{\infty}$, then (6.3) gives $\left\|\varphi_{j}\right\|_{\infty} \lesssim \delta^{-2}\left\|g_{j}\right\|_{\infty}$, and therefore $\varphi_{j}$ is bounded on $\mathbb{D}$. Also, (6.3) and an easy computation gives

$$
\left|\nabla \varphi_{j}(z)\right| \lesssim \delta^{-3} \sum_{l=1}^{n}\left|g_{l}^{\prime}(z)\right|
$$

Then, since $\left|g_{l}^{\prime}(z)\right|^{p} d A_{p, s}(z)$ is an $(s, p)$-Carleson measure, it follows that the measure $\left|\nabla \varphi_{j}(z)\right|^{p} d A_{p, s}(z)$ is an $(s, p)$-Carleson measure, and the proof is complete.

Let $g=\left(g_{1}, \ldots, g_{n}\right) \in H(\mathbb{D})^{n}$, and $f=\left(f_{1}, \ldots, f_{n}\right) \in H(\mathbb{D})^{n}$, and consider the operator $M_{g}$ defined by

$$
M_{g}(f)=\sum_{k=1}^{n} f_{k} g_{k}
$$

As an immediate consequence of Theorem 6.5 we obtain the following corona type decomposition. Note that the case $p=2$ was proved in [31].

Corollary 6.6. Let $1<p<\infty$ and $0<s<1$. The following are equivalent:

(i) $M_{g}$ maps $M\left(B_{p}(s)\right) \times \cdots \times M\left(B_{p}(s)\right)$ boundedly onto $M\left(B_{p}(s)\right)$;

(ii) $M_{g}$ maps $B_{p}(s) \times \cdots \times B_{p}(s)$ boundedly onto $B_{p}(s)$;

(iii) $g_{1}, \ldots, g_{n} \in M\left(B_{p}(s)\right)$ and (6.3) holds. 
Proof. (i) implies (ii). Since $M_{g}$ maps $M\left(B_{p}(s)\right)^{n}$ into $M\left(B_{p}(s)\right)$ it follows that $g_{1}, \ldots g_{n} \in M\left(B_{p}(s)\right)$. Then, if $f_{1}, \ldots, f_{n} \in B_{p}(s), M_{g}(f) \in B_{p}(s)$, and it follows from the closed graph theorem that $M_{g}$ maps $B_{p}(s) \times \cdots \times B_{p}(s)$ into $B_{p}(s)$. Now, let $h \in B_{p}(s)$. Then, by $(i)$, there are functions $h_{1}, \ldots, h_{n} \in M\left(B_{p}(s)\right)$ such that $\sum g_{j} h_{j}=1$. Then the functions $f_{k}=h_{k} h$ are in $B_{p}(s)$ and $\sum f_{k} g_{k}=h$. Thus, $M_{g}$ is surjective.

(ii) implies (iii). It is clear that if $M_{g}$ maps $B_{p}(s) \times \cdots \times B_{p}(s)$ into $B_{p}(s)$, then the functions $g_{j}$ are pointwise multipliers of $B_{p}(s)$. To see that (6.3) holds, let $f \in B_{p}(s)$. Since $M_{g}$ is onto, then it follows from the open mapping theorem that there exist functions $f_{j} \in B_{p}(s)$ with $f=\sum_{j=1}^{n} f_{j} g_{j}$ and $\left\|f_{j}\right\|_{B_{p}(s)} \leq C\|f\|_{B_{p}(s)}$. Now, using that $\left|f_{j}(z)\right| \leq\left\|f_{j}\right\|_{B_{p}(s)}\left\|k_{z}\right\|_{B_{q}(s)}$, we obtain

$$
|f(z)| \leq \sum_{j=1}^{n}\left|f_{j}(z)\right|\left|g_{j}(z)\right| \leq C\left\|k_{z}\right\|_{B_{q}(s)} \sum_{j=1}^{n}\left|g_{j}(z)\right| .
$$

Taking the function $f_{z}(w)=\frac{\left(1-|w|^{2}\right)^{s / p}}{(1-\bar{z} w)^{2 s / p}}$ that is in $B_{p}(s)$, with $\left\|f_{z}\right\|_{B_{p}(s)} \leq C$ (just use Lemma A to see that), and since $\left\|k_{z}\right\|_{B_{q}(s)} \asymp\left(1-|z|^{2}\right)^{-s / p}$, then (6.5) gives

$$
\sum_{j=1}^{n}\left|g_{j}(z)\right| \geq C>0
$$

proving (6.3).

(iii) implies (i). By Theorem 6.5, there exist functions $h_{1}, \ldots, h_{n} \in M\left(B_{p}(s)\right)$ with $\sum_{k=1}^{n} h_{k} g_{k}=1$. Then, if $f \in M\left(B_{p}(s)\right)$, the functions $f_{k}=f h_{k}$ are in $M\left(B_{p}(s)\right)$. Hence $M_{g}$ is surjective.

\section{SufficienCy of CONDition (CS)}

(CS) $\Rightarrow$ (UIS). Given a separated sequence $Z=\left\{z_{n}\right\}$ with $\mu_{Z}=\sum_{n}\left(1-\left|z_{n}\right|^{2}\right)^{s} \delta_{z_{n}}$ an $(s, p)$-Carleson measure. We must show that the map

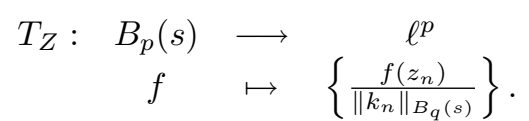

is bounded and onto. Since $\mu_{Z}$ is an $(s, p)$-Carleson measure,

$$
\left\|\frac{f\left(z_{n}\right)}{\left\|k_{n}\right\|_{B_{q}(s)}}\right\|_{\ell^{p}}^{p} \asymp \sum_{n}\left|f\left(z_{n}\right)\right|^{p}\left(1-\left|z_{n}\right|^{2}\right)^{s}=\int_{\mathbb{D}}|f(z)|^{p} d \mu_{Z}(z) \lesssim\|f\|_{B_{p}(s)}^{p} .
$$

To see that $T_{Z}$ is also onto, consider an arbitrary sequence $\left\{w_{n}\right\} \in l^{p}$. We will find a function $f \in B_{p}(s)$ with

$$
f\left(z_{n}\right)=w_{n}\left\|k_{n}\right\|_{B_{q}(s)} \quad \text { for } \quad n=1,2, \ldots
$$

By a standard normal family argument, it suffices to consider a finite sequence $z_{1}, \ldots, z_{N}$, provided we have uniform control on the norm of the interpolating functions.

Since $\left\{z_{n}\right\}$ is separated, there exists $\varepsilon>0$ such that the pseudohyperbolic discs $D\left(z_{n}, 2 \varepsilon\right)$ are pairwise disjoints. With standard arguments, we construct a smooth function $\varphi$ such that $\varphi(z)=w_{n}\left\|k_{n}\right\|_{B_{q}(s)}$ for $z \in D\left(z_{n}, \varepsilon\right)$; $\varphi$ vanishes outside 
$\bigcup_{n} D\left(z_{n}, 2 \varepsilon\right)$, and $(1-|z|)|\nabla \varphi(z)| \leq\left|w_{n}\right|\left\|k_{n}\right\|_{B_{q}(s)}$ for all $z \in D\left(z_{n}, 2 \varepsilon\right)$. The function $\varphi$ solves the interpolation problem with the data required, but it is not analytic. To arrange that we will solve an appropriate $\bar{\partial}$-problem. Using the above conditions on $\varphi$, we get

$$
\begin{aligned}
\int_{\mathbb{D}}|\nabla \varphi(z)|^{p} d A_{p, s}(z) & \leq \sum_{n} \int_{D\left(z_{n}, 2 \varepsilon\right)}\left|w_{n}\right|^{p}\left\|k_{n}\right\|_{B_{q}(s)}^{p}(1-|z|)^{s-2} d A(z) \\
& \lesssim \sum_{n}\left|w_{n}\right|^{p}<\infty
\end{aligned}
$$

Let $B(z)$ be the Blaschke product with zeros $\left\{z_{n}\right\}$. By (3.2), $\sum\left(1-\left|z_{n}\right|\right) \delta_{z_{n}}$ is a Carleson measure, and there exists a constant $C>0$ such that $|B(z)| \geq C$ for all $z$ in the support of $\bar{\partial} \varphi$. Then $\bar{\partial} \varphi / B \in \mathcal{C}(\overline{\mathbb{D}})$. So, by Theorem 6.2 , we can find a solution $u$ of the $\bar{\partial}$-equation $\bar{\partial} u=\frac{1}{B} \bar{\partial} \varphi$ with boundary values in $L_{s}^{p}$ and

$$
\|u\|_{L_{s}^{p}}^{p} \leq C \int_{\mathbb{D}}\left|\frac{\bar{\partial} \varphi(z)}{B(z)}\right|^{p} d A_{p, s}(z) \leq C\|\mid \nabla \varphi\|_{L^{p}\left(d A_{p, s}\right)}^{p} .
$$

Then the function $f=\varphi-B u$ is analytic on $\mathbb{D}$, and solves the interpolation problem $f\left(z_{n}\right)=\varphi\left(z_{n}\right)=w_{n}\left\|k_{n}\right\|_{B_{q}(s)}$ for each $n$. So, it only remains to prove that $f \in B_{p}(s)$. By Corollary 4.3 , it is enough to see that $f$ has boundary values in $L_{s}^{p}$. By Proposition 3.2, the Blaschke product $B$ belongs to $M\left(B_{p}(s)\right)$, and so, $B u$ has boundary values in $L_{s}^{p}$ with $\|B u\|_{L_{s}^{p}} \leq C\|u\|_{L_{s}^{p}}$. Since the boundary values of $\varphi$ are identically zero, we can conclude that the function $f=\varphi-B u \in B_{p}(s)$ with

$$
\|f\|_{B_{p}(s)} \leq C\|b\|_{L_{s}^{p}} \leq C\left\|w_{n}\right\|_{l^{p}}
$$

for some constant $C$ not depending on the number of points $N$. Hence the proof is complete.

$(\mathbf{C S}) \Rightarrow(\mathbf{M})$. Let $Z$ be a separated sequence and $\mu_{Z}=\sum_{z_{n} \in Z}\left(1-\left|z_{n}\right|^{2}\right)^{s} \delta_{z_{n}}$ be an $(s, p)$-Carleson measure. We want to see that $Z$ is an interpolating sequence for $M\left(B_{p}(s)\right)$. Let $\left\{w_{n}\right\} \in \ell^{\infty}$. Since $Z=\left\{z_{n}\right\}$ is separated, there is $\varepsilon>0$ such that the pseudohyperbolic discs $D_{n}=D\left(z_{n}, 2 \varepsilon\right)$ are pairwise disjoints. As before, we construct a smooth function $\varphi$ with $0 \leq|\varphi| \leq 1$ on $\mathbb{D}$ such that $\varphi(z)=w_{n}$ for $z \in D\left(z_{n}, \varepsilon\right) ; \varphi$ vanishes outside $\bigcup_{n} D\left(z_{n}, 2 \varepsilon\right)$; and $(1-|z|)|\nabla \varphi(z)| \leq C$ for all $z \in \mathbb{D}$.

Now, assume for a moment that we have seen that $|\nabla \varphi(z)|^{p} d A_{p, s}(z)$ is an $(s, p)$ Carleson measure. Let $B$ be the Blaschke product with zeros $\left\{z_{n}\right\}$. Since $|B(z)| \geq$ $C$ for all $z$ in the support of $\bar{\partial} \varphi$, we have $|\bar{\partial} \varphi / B| \leq C|\nabla \varphi|$, and therefore

$$
\left|\frac{\bar{\partial} \varphi}{B}(z)\right|^{p} d A_{p, s}(z)
$$

is an $(s, p)$-Carleson measure. Hence, by Theorem 6.4 , there is a solution $u$ of the $\bar{\partial}$-problem

$$
\bar{\partial} u=\frac{1}{B} \bar{\partial} \varphi
$$

with boundary values in $M\left(L_{s}^{p}\right)$. Then the function $g=\varphi-B u$ is analytic on $\mathbb{D}$, and $g\left(z_{n}\right)=\varphi\left(z_{n}\right)=w_{n}$ solving the interpolation problem with the data required. To see that the function $g \in M\left(B_{p}(s)\right)$, by Corollary 4.7, it is enough to show that $g$ has boundary values $M\left(L_{s}^{p}\right)$. By Lemma 3.2, the Blaschke product $B \in M\left(B_{p}(s)\right)$, and therefore, $B u$ has boundary values in $M\left(L_{s}^{p}\right)$. Since the boundary values of $\varphi$ 
are identically zero, we deduce that $g=\varphi-B u$ belongs to $M\left(B_{p}(s)\right)$ finishing the proof.

So, it only remains to show that $|\nabla \varphi(z)|^{p} d A_{p, s}(z)$ is an $(s, p)$-Carleson measure. Let $f \in B_{p}(s)$. Since $\bar{\partial} \varphi$ is supported on $\bigcup D_{n}$ and $(1-|z|)|\nabla \varphi(z)| \leq C$, we have

$$
\begin{aligned}
\int_{\mathbb{D}}|f(z)|^{p} & |\nabla \varphi(z)|^{p} d A_{p, s}(z) \leq \sum_{n} \int_{D_{n}}|f(z)|^{p}\left(1-|z|^{2}\right)^{s-2} d A(z) \\
& \lesssim \sum_{n}\left|f\left(z_{n}\right)\right|^{p}\left(1-\left|z_{n}\right|^{2}\right)^{s} \\
& +\sum_{n} \int_{D_{n}}\left|f(z)-f\left(z_{n}\right)\right|^{p}\left(1-|z|^{2}\right)^{s-2} d A(z) .
\end{aligned}
$$

Since $\sum_{n}\left(1-\left|z_{n}\right|^{2}\right)^{s} \delta_{z_{n}}$ is an $(s, p)$-Carleson measure, we have

$$
\sum_{n}\left|f\left(z_{n}\right)\right|^{p}\left(1-\left|z_{n}\right|^{2}\right)^{s} \leq C\|f\|_{B_{p}(s)}^{p} .
$$

So, if we prove

$$
\sum_{n} \int_{D_{n}}\left|f(z)-f\left(z_{n}\right)\right|^{p}\left(1-|z|^{2}\right)^{s-2} d A(z) \leq C\|f\|_{B_{p}(s)}^{p},
$$

then, joining (7.1), (7.2) and (7.3), we obtain

$$
\int_{\mathbb{D}}|f(z)|^{p}|\nabla \varphi(z)|^{p} d A_{p, s}(z) \lesssim\|f\|_{B_{p}(s)}^{p}
$$

proving that the measure $|\nabla \varphi(z)|^{p} d A_{p, s}(z)$ is an $(s, p)$-Carleson measure. To see (7.3), we use the reproducing formula (1.1), and inequality (2.2), to obtain

$$
\left|f(z)-f\left(z_{n}\right)\right| \lesssim \int_{\mathbb{D}}\left|f^{\prime}(w)\right| \frac{\left|z-z_{n}\right|}{|1-\bar{w} z|^{2+s}}\left(1-|w|^{2}\right)^{s} d A(w) .
$$

Choose $t>0$ with $\max (0,2-p)<t p<1+s \cdot \min (1, p-1)$. Then, by Hölder's inequality and Lemma A

$$
\begin{aligned}
\left|f(z)-f\left(z_{n}\right)\right|^{p} \lesssim & \left(1-|z|^{2}\right)^{p}\left(\int_{\mathbb{D}}\left|f^{\prime}(w)\right|^{p} \frac{\left(1-|w|^{2}\right)^{t p}}{|1-\bar{w} z|^{2+s}} d A_{p, s}(w)\right) \\
& \cdot\left(\int_{\mathbb{D}} \frac{\left(1-|w|^{2}\right)^{-t q}}{|1-\bar{w} z|^{2+s}} d A_{q, s}(w)\right)^{p-1} \\
\lesssim & \left(\int_{\mathbb{D}}\left|f^{\prime}(w)\right|^{p} \frac{\left(1-|w|^{2}\right)^{t p}}{|1-\bar{w} z|^{2+s}} d A_{p, s}(w)\right)\left(1-|z|^{2}\right)^{2-t p}
\end{aligned}
$$

Hence, by Fubini's theorem and Lemma A we obtain

$$
\begin{aligned}
\sum_{n} \int_{D_{n}} & \left|f(z)-f\left(z_{n}\right)\right|^{p}\left(1-|z|^{2}\right)^{s-2} d A(z) \\
& \lesssim \sum_{n} \int_{D_{n}}\left(\int_{\mathbb{D}}\left|f^{\prime}(w)\right|^{p} \frac{\left(1-|w|^{2}\right)^{t p}}{|1-\bar{w} z|^{2+s}} d A_{p, s}(w)\right)\left(1-|z|^{2}\right)^{s-t p} d A(z) \\
& \leq \int_{\mathbb{D}}\left|f^{\prime}(w)\right|^{p}\left(\int_{\mathbb{D}} \frac{\left(1-|z|^{2}\right)^{s-t p}}{|1-\bar{w} z|^{2+s}} d A(z)\right)\left(1-|w|^{2}\right)^{t p} d A_{p, s}(w) \\
& \lesssim \int_{\mathbb{D}}\left|f^{\prime}(w)\right|^{p} d A_{p, s}(w) \leq\|f\|_{B_{p}(s)}^{p}
\end{aligned}
$$


Note that the conditions on $t$ ensures that the application of Lemma A is correct. So, the proof is complete.

Another construction of the interpolating function. In [12], Earl give a constructive proof of Carleson interpolating theorem for bounded analytic functions. Given a sequence $\left\{z_{n}\right\}$ in the unit disc with

$$
\inf _{k} \prod_{j: j \neq k} \rho\left(z_{j}, z_{k}\right):=\delta>0
$$

and a sequence $a=\left\{a_{j}\right\} \in \ell^{\infty}$, Earl constructed a Blaschke product $B$ with zeros $\left\{\zeta_{j}\right\}$ such that the function $f(z)=K\|a\|_{\ell^{\infty}} B(z)$ solves the interpolating problem

$$
f\left(z_{j}\right)=a_{j}, \quad j=1,2, \ldots,
$$

for some constant $K$. Also, the zeros $\left\{\zeta_{j}\right\}$ of the Blaschke product $B$ satisfy

$$
\rho\left(z_{j}, \zeta_{j}\right) \leq \delta / 3
$$

So, in view of Proposition 3.2, we can use the same construction to give a different proof of the implication (CS) $\Rightarrow(\mathrm{M})$ of Theorem 1 .

Theorem 7.1. Let $\left\{z_{n}\right\}$ be a separated sequence such that $\sum\left(1-\left|z_{n}\right|^{2}\right)^{s} \delta_{z_{n}}$ is an $(s, p)$-Carleson measure. Then, given $a=\left\{a_{n}\right\} \in \ell^{\infty}$, there is a constant $K$ and $a$ Blaschke product $B$ in the multiplier algebra $M\left(B_{p}(s)\right)$ such that the function

$$
f(z)=K\|a\|_{\ell^{\infty}} B(z)
$$

solves the interpolation problem $f\left(z_{j}\right)=a_{j} \quad j=1,2 \ldots$

Proof. Since $\sum\left(1-\left|z_{n}\right|^{2}\right)^{s} \delta_{z_{n}}$ is an $(s, p)$-Carleson measure, then $\sum\left(1-\left|z_{n}\right|^{2}\right) \delta_{z_{n}}$ is a 1-Carleson measure (see the first paragraphs of the proof of Proposition 3.2). This and the separation condition gives (7.4) for some $\delta>0$. Hence, by Earl's construction, there is a constant $K$ and a Blaschke product $B$ with zeros $\left\{\zeta_{j}\right\}$ such that the function $f(z)=K\|a\|_{\ell^{\infty}} B(z)$ interpolates the data required. To see that $B$ is a multiplier of $B_{p}(s)$, by Proposition 3.2 it is enough to prove that if $\left\{\zeta_{j}\right\}$ is a sequence of points in the unit disc with $\rho\left(z_{j}, \zeta_{j}\right)<\delta / 3$ for all $j$, then the measure $\nu=\sum\left(1-\left|\zeta_{j}\right|^{2}\right)^{s} \delta_{\zeta_{j}}$ is also an $(s, p)$-Carleson measure. To see that, let $g \in B_{p}(s)$, then

$$
\begin{aligned}
\sum_{n}\left|g\left(\zeta_{n}\right)\right|^{p} & \left(1-\left|\zeta_{n}\right|^{2}\right)^{s} \\
& \lesssim \sum_{n}\left|g\left(\zeta_{n}\right)-g\left(z_{n}\right)\right|^{p}\left(1-\left|\zeta_{n}\right|^{2}\right)^{s}+\sum_{n}\left|g\left(z_{n}\right)\right|^{p}\left(1-\left|\zeta_{n}\right|^{2}\right)^{s} \\
& =S_{1}+S_{2} .
\end{aligned}
$$

Since $\rho\left(z_{n}, \zeta_{n}\right)<\delta / 3<1$, then $\left(1-\left|\zeta_{n}\right|^{2}\right) \asymp\left(1-\left|z_{n}\right|^{2}\right)$, and since $\sum_{n}\left(1-\left|z_{n}\right|^{2}\right)^{s}$ is an $(s, p)$-Carleson measure we get

$$
S_{2} \lesssim \sum_{n}\left|g\left(z_{n}\right)\right|^{p}\left(1-\left|z_{n}\right|^{2}\right)^{s} \lesssim\|g\|_{B_{p}(s)}^{p}
$$


To estimate $S_{1}$, note that the subharmonicity of $\left|g^{\prime}\right|$ gives

$$
\begin{aligned}
\left|g\left(\zeta_{n}\right)-g\left(z_{n}\right)\right| & \leq \int_{\left[z_{n}, \zeta_{n}\right]}\left|g^{\prime}(w)\right||d w| \\
& \lesssim \int_{\left[z_{n}, \zeta_{n}\right]}\left(\frac{1}{(1-|w|)^{2}} \int_{D_{w}}\left|g^{\prime}(z)\right| d A(z)\right)|d w|,
\end{aligned}
$$

where $D_{w}=\left\{z: \rho(z, w)<\frac{\delta}{6}\right\}$. Since $\rho\left(z_{n}, \zeta_{n}\right)<\delta / 3$, then $D_{w} \subset D_{n}$ for each $w \in\left[z_{n}, \zeta_{n}\right]$, where $D_{n}=\left\{z: \rho\left(z, z_{n}\right)<\frac{\delta}{2}\right\}$. Note that the pseudohyperbolic discs $D_{n}$ are pairwise disjoints. Also one has $(1-|w|) \asymp\left(1-\left|z_{n}\right|\right)$. These facts, together with (7.7) gives

$$
\left|g\left(\zeta_{n}\right)-g\left(z_{n}\right)\right| \lesssim \frac{\left|z_{n}-\zeta_{n}\right|}{\left(1-\left|z_{n}\right|\right)^{2}} \int_{D_{n}}\left|g^{\prime}(z)\right| d A(z) .
$$

Since $\left|z_{n}-\zeta_{n}\right| \lesssim\left(1-\left|z_{n}\right|^{2}\right)$, then (7.8) and Hölder's inequality gives

$$
\begin{aligned}
S_{1} & \lesssim \sum_{n}\left(\int_{D_{n}}\left|g^{\prime}(z)\right| d A(z)\right)^{p}\left(1-\left|z_{n}\right|^{2}\right)^{s-p} \\
& \leq \sum_{n}\left(\int_{D_{n}}\left|g^{\prime}(z)\right|^{p} d A(z)\right)\left|D_{n}\right|^{p-1}\left(1-\left|z_{n}\right|^{2}\right)^{s-p} \\
& \lesssim \sum_{n} \int_{D_{n}}\left|g^{\prime}(z)\right|^{p} d A_{p, s}(z) \leq\|g\|_{B_{p}(s)}^{p} .
\end{aligned}
$$

So, putting together (7.6) and (7.9), we obtain that $\nu$ is an $(s, p)$-Carleson measure, and this concludes the proof.

\section{Concluding Remarks}

Using the characterization of interpolating sequences obtained in Theorem 1, together with the description of Carleson measures for the spaces $B_{p}(s)$ obtained in [1] and [30], one can give explicit examples of interpolating sequences. For example, one has that a radial sequence is interpolating if and only if it is separated. It also follows that different values of $s$ give different classes of interpolating sequences.

Here are some questions for which we do not have a ready answer.

(1) By retracing the steps of the proof, it can be seen that the solution of the interpolating problem is an explicit operator which is not linear with respect to the data. Is there a linear interpolating operator? See [6].

(2) Characterize the interpolating sequences for a larger class of weighted Besov spaces, where the weights are not necessarily of the form $\left(1-|z|^{2}\right)^{s}$. A related problem is that of characterizing the interpolating sequences for spaces with the complete Nevanlinna-Pick property. See [23].

\section{REFERENCES}

[1] N. Arcozzi, R. Rochberg, E. Sawyer, Carleson measures for analytic Besov spaces, Rev. Mat. Iberoamericana 18 (2002), 443-510.

[2] N. Arcozzi, R. Rochberg, E. Sawyer, Carleson Measures and Interpolating Sequences for Besov Spaces on Complex Balls, Memoirs Amer. Math. Soc. 859 (2006).

[3] C.J. Bishop, Interpolating sequences for the Dirichlet space and its multipliers, Manuscript, 1994.

[4] O. Blasco, Operators on weighted Bergman spaces $(0<p \leq 1)$ and applications, Duke Math. J. 66 (1992), 443-467. 
[5] D. Blasi, J. Pau, A characterization of Besov type spaces and applications to Hankel type operators, Michigan Math. J., (2008), to appear.

[6] B. Böe, Interpolating sequences for Besov spaces, J. Funct. Anal. 192 (2002), 319-341.

[7] B. Böe, An interpolation theorem for Hilbert spaces with Nevanlinna-Pick kernel, Proc. Amer. Math. Soc. 133 (2005), 2077-2081.

[8] L. Carleson, An interpolation problem for bounded analytic functions, Amer. J. Math. 80 (1958), 921-930.

[9] L. Carleson, Interpolation by bounded analytic functions and the corona problem, Ann. of Math. 76 (1962), 547-559.

[10] R. Chartrand, Multipliers and Carleson measures for $D(\mu)$, Integral Equations Operator Theory 45 (2003), 309-318.

[11] W. Cohn, Interpolation and multipliers on Besov and Sobolev spaces, Complex Variables 22 (1993), 35-45.

[12] J.P. Earl, On the interpolation of bounded sequences by bounded functions, J. London Math. Soc. 2 (1970), 544-548.

[13] J.B. Garnett, Bounded Analytic Functions, Academic Press, New York, 1981.

[14] H. Hedenmalm, B. Korenblum, K. Zhu, Theory of Bergman spaces, Graduate Texts in Mathematics 199, Springer-Verlag, New York, 2000.

[15] L. Hörmander, Generators for some rings of analytic functions, Bull. Amer. Math. Soc. 73 (1967), 943-949.

[16] P.W. Jones, $L^{\infty}$ estimates for the $\bar{\partial}$ problem in a half-plane, Acta Math. 150 (1983), 137-152.

[17] V. Kabaila, Interpolation sequences for the $H_{p}$ classes in the case $p<1$, Litovsk. Mat. Sb. 3 (1963), 141-147 (Russian).

[18] R. Kerman, E. Sawyer, Carleson measures and multipliers of Dirichlet-type spaces, Trans. Amer. Math. Soc. 309 (1988), 87-98.

[19] D.E. Marshall, C. Sundberg, Interpolating sequences for the multipliers of the Dirichlet space, Manuscript 1994. Avalaible at http://www.math.washington.edu/marshall/ preprints/preprints.html

[20] A. Nicolau, The corona property for bounded analytic functions in some Besov spaces, Proc. Amer. Math. Soc. 110 (1990), 135-140.

[21] A. Nicolau, J. Xiao, Bounded functions in Möbius invariant Dirichlet spaces, J. Funct. Anal. 150 (1997), 383-425.

[22] R. Rochberg, Z. Wu, A new characterization of Dirichlet type spaces and applications, Illinois J. Math. 37 (1993), 101-122.

[23] K. Seip, Interpolation and Sampling in spaces of analytic functions, University Lecture Series 33, AMS, Providence, 2004.

[24] H.S. Shapiro, A.L. Shields, On some interpolation problems for analytic functions, Amer. J. Math. 83 (1961), 513-532.

[25] D.A. Stegenga, Multipliers of Dirichlet spaces, Illinois J. Math. 24 (1980), 113-139.

[26] E.M. Stein, Singular integrals and differentiability properties of functions, Princeton University Press, Princeton, N.J. 1970.

[27] V.A. Tolokonnikov, The corona theorem in algebras of bounded analytic functions, Amer. Math. Soc. Trans. 149 (1991), 61-93.

[28] N. Varopoulos, Sur un problème d'interpolation, C.R. Acad. Sci. Paris, Sér. A 274 (1972), $1539-1542$.

[29] I.E. Verbitsky, Multipliers in spaces with "fractional" norms, and inner functions, Sibirsk. Math. Zh. 26 (1985), 51-72.(Russian)

[30] Z. Wu, Carleson measures and multipliers for Dirichlet spaces, J. Funct. Anal. 169 (1999), 148-163.

[31] J. Xiao, The $\bar{\partial}$-problem for multipliers of the Sobolev space, Manuscripta Math. 97 (1998), $217-232$.

[32] K. Zhu, Operator Theory in Function Spaces, Marcel Dekker, New York, 1990. 
Department of Mathematics, University of Bologna, Piazza di Porta San Donato, 5 , 40127 BoLOGNA, ITALY

E-mail address: arcozzi@dm.unibo.it

Departament de Matemàtiques, Universitat Autònoma de Barcelona, 08193 Bellaterra, SPAIN

E-mail address: dblasi@mat.uab.cat

Departament de Matemàtica Aplicada i Analisi, Universitat de Barcelona, Gran Via de les Corts Catalanes, 585, 08007 Barcelona, SPAIN

E-mail address: jordi.pau@ub.edu 